Pacific Journal of Mathematics

A COMPARISON ALGEBRA ON A CYLINDER WITH
SEMI-PERIODIC MULTIPLICATIONS 


\title{
A COMPARISON ALGEBRA ON A CYLINDER WITH SEMI-PERIODIC MULTIPLICATIONS
}

\author{
Severino T. Melo
}

\begin{abstract}
A necessary and sufficient Fredholm criterion is found for a $C^{*}$ algebra of bounded operators on a cylinder, which contains operators of the form $L \Lambda^{M}$, where $\Lambda=(1-\Delta)^{-1 / 2}$ and $L$ is an $M$ th order differential operator whose coefficients are periodic at infinity.
\end{abstract}

0. Introduction. Let $\Omega$ denote the cylinder $\mathbb{R} \times \mathbb{B}$, where $\mathbb{B}$ is a compact Riemannian manifold, $\Delta_{\Omega}$ its Laplacian and $\mathscr{H}$ the Hilbert space $L^{2}(\Omega)$. Cordes [3] found a necessary and sufficient Fredholm criterion for operators in the $C^{*}$-subalgebra of $\mathscr{L}(\mathscr{H})$ generated by: (i) multiplications by functions that extend continuously to $[-\infty,+\infty] \times \mathbb{B}$, (ii) $\Lambda=\left(1-\Delta_{\Omega}\right)^{-1 / 2}$ and (iii) operators of the form $D \Lambda$, where $D$ is either $\partial / \partial t, t \in \mathbb{R}$, or a first order differential operator on $\mathbb{B}$ with smooth coefficients. Here we extend this algebra by adjoining the multiplications by $2 \pi$-periodic continuous functions to the generators, and a similar Fredholm criterion is obtained.

The commutator ideal $\mathscr{E}_{\mathscr{P}}$ of the extended algebra $\mathscr{C}_{\mathscr{P}}$ is proven to be $*$-isomorphic to $\mathscr{S} \mathscr{L} \bar{\otimes} \mathscr{K}_{\mathbb{Z}} \bar{\otimes} \mathscr{K}_{\mathbb{B}}$, where $\mathscr{S} \mathscr{L}$ denotes the algebra of singular integral operators on the circle and $\mathscr{K}_{\mathbb{Z}}$ and $\mathscr{K}_{\mathbb{B}}$ denote the algebras of compact operators on $L^{2}(\mathbb{Z})$ and $L^{2}(\mathbb{B})$, respectively. This allows us to define on $\mathscr{C}_{\mathscr{P}}$ an operator-valued symbol, the " $\gamma$ symbol", such that $\operatorname{ker} \gamma \cap \operatorname{ker} \sigma$ equals the compact ideal of $\mathscr{L}(\mathscr{H})$. Here $\sigma$ denotes the complex-valued symbol on $\mathscr{C}_{\mathscr{P}}$ that arises from the Gelfand map of the commutative $C^{*}$-algebra $\mathscr{C}_{\mathscr{P}} / \mathscr{C}_{\mathscr{P}}$. We prove that $A \in \mathscr{C}_{\mathscr{P}}$ is Fredholm if and only if $\gamma_{A}$ and $\sigma_{A}$ are invertible.

The simpler case when the compact manifold reduces to a point is considered in [5]. There, a unitary map $W$ from $L^{2}(\mathbb{R})$ onto $L^{2}\left(S^{1}\right) \bar{\otimes} L^{2}(\mathbb{Z})$ is defined, such that the conjugate $W \mathscr{E} W^{-1}$ of the commutator ideal equals $\mathscr{S} \mathscr{L} \bar{\otimes} \mathscr{K}_{\mathbb{Z}}$. Here, we conjugate $\mathscr{E}_{\mathscr{P}}$ with $W \otimes I_{\mathbb{B}}$, where $I_{\mathbb{B}}$ denotes the identity operator on $L^{2}(\mathbb{B})$, and obtain $\mathscr{S} \mathscr{L} \otimes \mathscr{K}_{\mathbb{Z}} \bar{\otimes} \mathscr{K}_{\mathbb{B}}$.

If $L$ is a differential operator on $\Omega$ whose coefficients are continuous and approach periodic functions at infinity, the operator $A=$ $L \Lambda^{M}$ belongs to $\mathscr{C}_{\mathscr{P}}$, where $M$ is the order of $L$. We can apply 
the criterion above to $A$ and prove that $L$ is a Fredholm operator if and only if it is uniformly elliptic and a certain family of elliptic differential operators on the compact manifold $S^{1} \times \mathbb{B}$ is invertible. This applies also for matrices of such operators.

These results can be extended in a standard way to non-compact manifolds with cylindrical ends (cf. [2], VIII-3,4). Fredholm properties of elliptic-differential operators on such manifolds have been studied, for example, by Lockhart-McOwen [6] and Taubes [8]. The case where the coefficients are periodic on the ends is included in Taubes' results.

\section{Definition of the algebra $\mathscr{C}_{\mathscr{P}}$ and a description of its commutator} ideal. Let $\Omega$ denote the Riemannian manifold $\mathbb{R} \times \mathbb{B}$, where $\mathbb{B}$ denotes an $n$-dimensional compact manifold with metric tensor locally given by $h_{j k}$, and let $\mathscr{H}$ denote the Hilbert space $L^{2}(\Omega)$, with $\Omega$ being given the surface measure

$$
d S=\sqrt{h} d t d x^{1} \cdots d x^{n},
$$

where $h$ is the determinant of the $n \times n$-matrix $\left(\left(h_{j k}\right)\right)_{1 \leq j, k \leq n}$. The metric on $\Omega$ is given by $d s^{2}=d t^{2}+h_{j k} d x^{j} d x^{k}$, and the Laplace operator is locally given by

$$
\Delta_{\Omega}=\Delta_{\mathbb{R}}+\Delta_{\mathbb{B}}=\frac{d^{2}}{d t^{2}}+\frac{1}{\sqrt{h}} \frac{\partial}{\partial x^{j}} \sqrt{h} h^{j k} \frac{\partial}{\partial x^{k}},
$$

where $\left(\left(h^{j k}\right)\right)=\left(\left(h_{j k}\right)\right)^{-1}$, and the summation convention from 1 to $n$ is being used.

The symmetric operator $\Delta_{\Omega}$ with domain $\mathbf{C}_{0}^{\infty}(\Omega)$ is essentially selfadjoint, since $\Omega$ is complete (cf. [2], IV). We denote by $H$ the closure of $1-\Delta_{\Omega}$ and by $\Lambda$ its inverse square root, $\Lambda=H^{-1 / 2}$. Since $H \geq 1$, we have $\Lambda \in \mathscr{L}(\mathscr{H})$. The algebra $\mathscr{C}_{\mathscr{P}}$ is defined as the smallest $C^{*}$ subalgebra of $\mathscr{L}(\mathscr{H})$ containing the following operators (or classes of operators):

$$
\begin{gathered}
a \in \mathbf{C}^{\infty}(\mathbb{B}) ; \quad b \in \mathbf{C S}(\mathbb{R}) ; \\
e^{i j t}, j \in \mathbb{Z} ; \quad \Lambda ; \quad \frac{1}{i} \frac{\partial}{\partial t} \Lambda \text { and } D_{x} \Lambda,
\end{gathered}
$$

$D_{x}$ being a first order differential operator on $\mathbb{B}$, locally given by $-i b^{j}(x) \partial / \partial x^{j}$, where $b^{j}(x), j=1, \ldots, n$, are the components of a smooth vector field on $\mathbb{B}$. The operators $\frac{\partial}{\partial t} \Lambda$ and $D_{x} \Lambda$, defined on the dense subspace $\Lambda^{-1}\left(C_{0}^{\infty}(\Omega)\right)$, can be extended to bounded operators of $\mathscr{L}(\mathscr{H})$ (cf. [2], for example). Bounded functions on $\Omega$ 
have been identified with the corresponding multiplication operators in $\mathscr{L}(\mathscr{H})$ and $\mathbf{C S}(\mathbb{R})$ denotes the class of continuous functions on $\mathbb{R}$ with limits at $+\infty$ and $-\infty$.

Our first objective is to obtain a necessary and sufficient criterion for an operator in $\mathscr{C}_{\mathscr{P}}$ to be Fredholm. Such a criterion has been found by Cordes [3] for the algebra generated by the operators in (1) except $e^{i j t}, j \in \mathbb{Z}$.

Taking advantage of the tensor product structure of $\mathscr{H}$,

$$
\mathscr{H}=L^{2}(\mathbb{R}) \bar{\otimes} L^{2}(\mathbb{B}),
$$

we consider the conjugate of $\mathscr{C}_{\mathscr{P}}$ with respect to the unitary operator $F \otimes I_{\mathbb{B}}$, where $I_{\mathbb{B}}$ denotes the identity operator on $L^{2}(\mathbb{B})$ and $F$ the Fourier transform on $L^{2}(\mathbb{R})$,

$$
(F u)(\tau)=\frac{1}{\sqrt{2 \pi}} \int e^{-i \tau t} u(t) d t .
$$

In order to simplify notation, $A \otimes I_{\mathbb{B}}$ is denoted by $A$ and $I_{\mathbb{R}} \otimes B$ by $B$, whenever $A \in \mathscr{L}\left(L^{2}(\mathbb{R})\right)$ or $B \in \mathscr{L}\left(L^{2}(\mathbb{B})\right)$.

We seek to describe what are $B_{k}:=F^{-1} A_{k} F$, where $A_{k}, k=$ $1, \ldots, 6$, denote the operators listed in $(1)$, in that order. The operatorvalued functions $\tilde{\Lambda}(\tau):=\left(1+\tau^{2}-\Delta_{\mathbb{B}}\right)^{-1 / 2}, \tau \tilde{\Lambda}(\tau)$ and $D_{x} \tilde{\Lambda}(\tau), \tau \in \mathbb{R}$, are all in $\mathbf{C B}\left(\mathbb{R}, \mathscr{L}_{\mathbb{B}}\right)$, as proven in [3], page 220, and thus determine operators in $\mathscr{L}(\mathscr{H})$ by multiplication in the real variable. Here $\mathscr{L}_{\mathbb{B}}$ denotes the algebra of bounded operators on $L^{2}(\mathbb{B})$ and $\mathbf{C B}\left(\mathbb{R}, \mathscr{L}_{\mathbb{B}}\right)$ the bounded continuous $\mathscr{L}_{\mathbb{B}}$-valued functions on $\mathbb{R}$. With this interpretation, we get $B_{k}, k=1, \ldots, 6$, respectively given by

$$
\begin{gathered}
a \in \mathbf{C}^{\infty}(\mathbb{B}) ; \quad b(D), b \in \mathrm{CS}(\mathbb{R}) ; \quad T_{j}, j \in \mathbb{Z} ; \\
\tilde{\Lambda}(\tau) ; \quad-\tau \tilde{\Lambda}(\tau) \quad \text { and } D_{x} \tilde{\Lambda}(\tau),
\end{gathered}
$$

where $b(D):=F^{-1} b F$ and $T_{j}$ denotes the translation $\left(T_{j} u\right)(\tau)=$ $u(\tau+j)$.

Let $\mathscr{K}_{\mathbb{B}}$ denote the ideal of compact operators on $L^{2}(\mathbb{B})$ and $\mathbf{C O}\left(\mathbb{R}, \mathscr{K}_{\mathbb{B}}\right)$ denote the $\mathscr{K}_{\mathbb{B}}$-valued continuous functions on $\mathbb{R}$ that vanish at infinity. All commutators $\left[B_{k}, B_{l}\right], k, l \neq 3$, are contained in the algebra

$$
\mathscr{C} \mathscr{K}:=\mathbf{C O}\left(\mathbb{R}, \mathscr{K}_{\mathbb{B}}\right)+\mathscr{K}(\mathscr{H}),
$$

where $\mathscr{K}(\mathscr{H})$ denotes the ideal of compact operators of $\mathscr{L}(\mathscr{H})$, as proven in [3], Proposition 1.2. Next we investigate what are the 
commutators $\left[B_{3}, B_{k}\right], k=1, \ldots, 6$. We easily get $\left[B_{3}, B_{1}\right]=$ $\left[B_{3}, B_{2}\right]=0$. It is also clear that, for any $K(\tau) \in \mathbf{C B}\left(\mathbb{R}, \mathscr{L}_{\mathbb{B}}\right)$, we have

$$
\left[T_{k}, K(\tau)\right]=(K(\tau+k)-K(\tau)) T_{k}, \quad k \in \mathbb{Z}
$$

PROPOSITION 1.1. The commutators of the generators in (2)-and of their adjoints-of the algebra $\widehat{C}_{\mathscr{P}}:=F^{-1 \mathscr{C}_{\mathscr{P}} F}$ are contained in $\mathscr{C} \mathscr{K T}=\left\{\sum_{j=-N}^{N} K_{j}(\tau) T_{j}+K ; N \in \mathbb{N}, K_{j} \in \mathbf{C O}(\mathbb{R}, \mathscr{K} \mathbb{B}), K \in \mathscr{H}(\mathscr{H})\right\}$.

Proof. Let us first prove that $K(\tau+j)-K(\tau) \in \mathbf{C O}\left(\mathbb{R}, \mathscr{H}_{\mathbb{B}}\right)$, for $K(\tau)=\tilde{\Lambda}(\tau), \tau \tilde{\Lambda}(\tau)$ or $D_{x} \tilde{\Lambda}(\tau)$. It follows from the fact that $-\Delta_{\mathbb{B}}$ on $L^{2}(\mathbb{B})$ has an orthonormal basis of eigenfunctions, with eigenvalues $0 \leq \lambda_{1} \leq \lambda_{2} \leq \cdots, \lambda_{n} \rightarrow \infty$ as $n \rightarrow \infty$, that, for each $\tau \in \mathbb{R}, \tilde{\Lambda}(\tau)$ is unitarily equivalent to the multiplication operator $\left(1+\tau^{2}+\lambda_{n}\right)^{-1 / 2}$ on $L^{2}(\mathbb{N})$. Hence: $\tilde{\Lambda}(\tau) \in \mathbf{C O}\left(\mathbb{R}, \mathscr{K}_{\mathbb{B}}\right)$,

$\|\tau[\tilde{\Lambda}(\tau+j)-\tilde{\Lambda}(\tau)]\|_{L^{2}(\mathbb{B})} \leq \max _{s \in[1, \infty)}\left|\tau\left[\left(s+(\tau+j)^{2}\right)^{-1 / 2}-\left(s+\tau^{2}\right)^{-1 / 2}\right]\right|$ and

$$
\left\|\tilde{\Lambda}(\tau)^{-1} \tilde{\Lambda}(\tau+j)-1\right\|_{L^{2}(\mathbb{R})} \leq \max _{s \in[1, \infty)}\left|\left(\tau^{2}+s\right)^{1 / 2}\left((\tau+j)^{2}+s\right)^{-1 / 2}-1\right| .
$$

Note that the right-hand sides of the two previous inequalities go to zero as $\tau \rightarrow \pm \infty$. Furthermore, as

$$
\lim _{n \rightarrow \infty}\left(1+\tau^{2}+\lambda_{n}\right)^{1 / 2}\left(1+(\tau+j)^{2}+\lambda_{n}\right)^{-1 / 2}-1=0,
$$

we have that $\tilde{\Lambda}(\tau)^{-1} \tilde{\Lambda}(\tau+j)-1 \in \mathscr{K}_{\mathbb{B}}$, for each $\tau \in \mathbb{R}$. We then get: $(\tau+j) \tilde{\Lambda}(\tau+j)-\tau \tilde{\Lambda}(\tau)=\tau(\tilde{\Lambda}(\tau+j)-\tilde{\Lambda}(\tau))+j \tilde{\Lambda}(\tau+j) \in \mathbf{C O}\left(\mathbb{R}, \mathscr{H}_{\mathbb{B}}\right)$ and

$$
D_{x} \tilde{\Lambda}(\tau+j)-D_{x} \tilde{\Lambda}(\tau)=D_{x} \tilde{\Lambda}(\tau)\left[\tilde{\Lambda}(\tau)^{-1} \tilde{\Lambda}(\tau+j)-1\right] \in \mathbf{C O}\left(\mathbb{R}, \mathscr{K}_{\mathbb{B}}\right)
$$

By the remarks preceding the statement of the proposition, this proves that the commutators of the generators (2) are indeed contained in $\mathscr{C} \mathscr{R}$. Concerning the adjoints, let us note that the classes of $B_{k}$ 's, $k=1, \ldots, 5$, are self-adjoint and that, as proven in [3], $D_{x} \tilde{\Lambda}-\tilde{\Lambda} D_{x} \in \mathbf{C O}\left(\mathbb{R}, \mathscr{K}_{\mathbb{B}}\right)$. Hence

$$
\left(D_{x} \tilde{\Lambda}\right)^{*}-D_{x}^{*} \tilde{\Lambda}=\tilde{\Lambda} D_{x}^{*}-D_{x}^{*} \tilde{\Lambda} \in \mathbf{C O}\left(\mathbb{R}, \mathscr{K}_{\mathbb{B}}\right) .
$$


Here, $D_{x}^{*}$ denotes the formal adjoint of $D_{x}$. The commutators of any $K(\tau) \in \mathbf{C O}\left(\mathbb{R}, \mathscr{K}_{\mathbb{B}}\right)$ with the generators $B_{k}, k=1,3,4,5,6$, are clearly contained in $\mathscr{C} \mathscr{R T}$. For $K(\tau)$ of the special form $K(\tau)=$ $a(\tau) \widetilde{K}, a \in \mathbf{C O}(\mathbb{R})$ and $\widetilde{K} \in \mathscr{K}_{\mathbb{B}}$, the commutator $[b(D), K(\tau)]=$ $[b(D), a(\tau)] \otimes \widetilde{K}$ is compact, since $[b(D), a(\tau)]$ is compact (cf. [4], Chapter III, for example), for $b \in \mathbf{C S}(\mathbb{R})$. The vector space generated by all $K(\tau)=a(\tau) \widetilde{K}$ as above is dense in $\operatorname{CO}\left(\mathbb{R}, \mathscr{K}_{\mathbb{B}}\right)$ and thus we have

$$
[b(D), K(\tau)] \in \mathscr{K}(\mathscr{H}), \quad \text { for } b \in \mathbf{C S}(\mathbb{R}), K(\tau) \in \mathbf{C O}\left(\mathbb{R}, \mathscr{K}_{\mathbb{B}}\right) .
$$

This concludes the proof.

Denoting by $\mathscr{E}_{\mathscr{P}}$ the commutator ideal of $\mathscr{C}_{\mathscr{P}}$ and by $\mathscr{E}_{\mathscr{P}}$ the commutator ideal of $\hat{\mathscr{C}}_{\mathscr{P}}$, it is obvious that $\hat{\mathscr{C}}_{\mathscr{P}}=F^{-1} \mathscr{E}_{\mathscr{P}} F$.

Proposition 1.2. The commutator ideal $\hat{\mathscr{E}}_{\mathscr{P}}$ of the algebra $\hat{\mathscr{C}}_{\mathscr{P}}$ is obtained by closing the set of operators:

$$
\begin{aligned}
\widehat{\mathscr{E}}_{P, 0}:= & \left\{\sum_{j=-N}^{N} b_{j}(D) K_{j}(\tau) T_{j}+K\right. \\
& b_{j} \in \mathbf{C S}(\mathbb{R}), N \in \mathbb{N}, K_{j} \in \mathbf{C O}(\mathbb{R}, \mathscr{H} \mathbb{B}, K \in \mathscr{K}(\mathscr{H})\} .
\end{aligned}
$$

Proof. The algebra $\mathscr{C}_{\mathscr{P}}$ is a "Comparison Algebra", in the sense of [2], Chapter V, with "generating classes":

$$
\mathscr{A}^{\sharp}:=\mathbf{C}_{0}^{\infty}(\Omega) \cup \mathbf{C}^{\infty}(\mathbb{B}) \cup\left\{e^{i j t} ; j \in \mathbb{Z}\right\} \cup\left\{s(t)=t\left(1+t^{2}\right)^{-1 / 2}\right\}
$$

and $\mathscr{D}^{\sharp}$ equal to the vector space generated by the first order linear partial differential expressions on $\mathbb{B}$ with smooth coefficients and by the expression $\partial / \partial t$. Indeed, $\mathscr{C}_{\mathscr{P}}$ can alternatively be defined as the $C^{*}$-algebra generated by all multiplications by functions in $\mathscr{A}^{\sharp}$ and by all $D \Lambda, D \in \mathscr{D}^{\sharp}$. It follows then from Lemma V-1-1 of [2] that $\mathscr{K}(\mathscr{H}) \subset \mathscr{E}_{\mathscr{P}}$ and therefore $\mathscr{K}(\mathscr{H}) \subset \hat{\mathscr{E}}_{\mathscr{P}}$. Moreover, it was proven in [3], Proposition 1.5 , that $\mathbf{C O}\left(\mathbb{R}, \mathscr{H}_{\mathbb{B}}\right)$ is contained in the commutator ideal of the $C^{*}$-algebra generated by $B_{4}, B_{5}$ and $B_{6}$. Thus we get $\widehat{\mathscr{E}}_{P, 0} \subset \widehat{\mathscr{E}}_{\mathscr{P}}$.

All commutators of the generators (2) and their adjoints are contained in $\widehat{\mathscr{E}}_{P, 0}$, by Proposition 1.1. Again using (3), (4) and (5), it is easy to verify that $\widehat{\mathscr{E}}_{P, 0}$ is invariant under right or left multiplication by the operators in (2) and their adjoints. Two facts then follow: 
(i) all commutators of the algebra (finitely) generated by the operators in (2) and their adjoints are contained in $\hat{\mathscr{E}}_{P, 0}$ and therefore all commutators of $\hat{\mathscr{C}}_{\mathscr{P}}$ are contained in the closure of $\hat{\mathscr{E}}_{P, 0}$, and (ii) the closure of $\hat{\mathscr{E}}_{P, 0}$ is an ideal of $\hat{\mathscr{C}}_{\mathscr{P}}$. By definition of commutator ideal, $\widehat{\mathscr{E}}_{\mathscr{P}}$ is contained in the closure of $\widehat{\mathscr{E}}_{P, 0}$.

Let $\mathbf{C O}(\mathbb{R})$ denote the set of continuous functions on $\mathbb{R}$ vanishing at infinity and let $\hat{\mathscr{E}}_{0}$ denote the set of bounded operators on $L^{2}(\mathbb{R})$

$$
\begin{aligned}
\widehat{\mathscr{E}}_{0}:=\left\{\sum_{j=-N}^{N} b_{j}(D) a_{j}(\tau) T_{j}+K ; N \in \mathbb{N}, b_{j}\right. & \in \mathbf{C S}(\mathbb{R}), \\
& \left.a_{j} \in \mathbf{C O}(\mathbb{R}), K \in \mathscr{H} \mathbb{R}\right\} .
\end{aligned}
$$

COROLlARY 1.3. With $\hat{\mathscr{E}}$ denoting the closure of $\hat{\mathscr{E}}_{0}$ defined above, we have:

$$
\widehat{\mathscr{E}}_{\mathscr{p}}=\widehat{\mathscr{E}} \bar{\otimes} \mathscr{K}_{\mathbb{B}}
$$

where $\bar{\otimes}$ denotes the operator-norm closure of the algebraic tensor product.

Proof. The vector-space generated by

$$
\left\{\left(b(D) a(\tau) T_{j}+K\right) \otimes \widetilde{K} ; b \in \mathbf{C S}(\mathbb{R}), a \in \mathbf{C O}(\mathbb{R}), j \in \mathbb{Z},\right.
$$

$$
\left.K \in \mathscr{K}_{\mathbb{R}}, \widetilde{K} \in \mathscr{K}_{\mathbb{B}}\right\}
$$

is dense in $\widehat{\mathscr{E}}_{P, 0}$ and in $\hat{\mathscr{E}} \bar{\otimes} \mathscr{K}_{\mathbb{B}}$.

In the rest of this section, we define a unitary map

$$
W: L^{2}(\mathbb{R}) \rightarrow L^{2}\left(\mathbb{S}^{1} ; L^{2}(\mathbb{Z})\right)
$$

and find a useful description for $\left(W \otimes I_{\mathbb{B}}\right) \widehat{\mathscr{E}}_{\mathscr{P}}\left(W \otimes I_{\mathbb{B}}\right)^{-1}$.

Given $u \in L^{2}(\mathbb{R})$, denote:

$$
u^{\diamond}(\varphi):=(u(\varphi-j))_{j \in \mathbb{Z}},
$$

for each $\varphi \in \mathbb{R}$. The sequence $u^{\diamond}(\varphi)$ belongs to $L^{2}(\mathbb{Z})$ for almost every $\varphi$, by Fubini's Theorem, since $L^{2}(\mathbb{R})$ can be identified with $L^{2}([0,1) \times \mathbb{Z})$. Let

$$
F_{d}: L^{2}\left(\mathbb{S}^{1}, d \theta\right) \rightarrow L^{2}(\mathbb{Z}), \quad \mathbb{S}^{1}=\left\{e^{i \theta} ; \theta \in \mathbb{R}\right\},
$$

denote the discrete Fourier transform:

$$
\left(F_{d} u\right)_{j}=\frac{1}{\sqrt{2 \pi}} \int_{0}^{2 \pi} u(\theta) e^{-i j \theta} d \theta, \quad j \in \mathbb{Z}
$$


For each $\varphi \in \mathbb{R}$, define

$$
Y_{\varphi}:=F_{d} e^{-i \varphi \theta} F_{d}^{-1}
$$

The operators $Y_{\varphi}$ define a smooth function on $\mathbb{R}$, taking values in the unitary operators on $L^{2}(\mathbb{Z})$ and satisfying $\left(Y_{k} u\right)_{j}=u_{j+k}$, for $k \in \mathbb{Z}$ and $u \in L^{2}(\mathbb{Z})$, and $Y_{\varphi} Y_{\omega}=Y_{\varphi+\omega}$, for $\varphi, \omega \in \mathbb{R}$.

We now define the map (with $\mathbb{S}^{1}=\left\{e^{2 \pi i \varphi} ; \varphi \in \mathbb{R}\right\}$ )

$$
\begin{aligned}
W: L^{2}(\mathbb{R}) & \rightarrow L^{2}\left(\mathbb{S}^{1}, d \varphi ; L^{2}(\mathbb{Z})\right), \\
u & \mapsto(W u)(\varphi)=Y_{\varphi} u^{\diamond}(\varphi) .
\end{aligned}
$$

Let $\mathbf{C S}(\mathbb{Z})$ denote the set of sequences $b(j), j \in \mathbb{Z}$, with limits as $j \rightarrow+\infty$ and $j \rightarrow-\infty$ and let $b\left(D_{\theta}\right)$ denote $F_{d}^{-1} b(M) F_{d}$, where $b(M)$ denotes the operator multiplication by $b$ on $L^{2}(\mathbb{Z})$. We then denote by $\mathscr{S} \mathscr{L}$ the $C^{*}$-subalgebra of $\mathscr{L}_{\mathbb{S}^{1}}:=\mathscr{L}\left(L^{2}\left(\mathbb{S}^{1}\right)\right)$ generated by $b\left(D_{\theta}\right), b \in \mathscr{C S}(\mathbb{Z})$, and by the multiplications by smooth functions on $\mathbb{S}^{1}$. It is easy to check that, with $\Lambda_{\mathbb{S}^{1}}:=\left(1-\Delta_{\mathbb{S}^{1}}\right)^{-1 / 2}$,

$$
\frac{1}{i} \frac{d}{d \theta} \Lambda_{\mathbb{S}^{1}}=s\left(D_{\theta}\right), \quad s(j)=\left(1+j^{2}\right)^{-1 / 2}
$$

Since the polynomials in $s$ are dense in $\mathbf{C S}(\mathbb{Z}), \mathscr{S} \mathscr{L}$ coincides with the $C^{*}$-subalgebra of $\mathscr{L}_{\mathbb{S}^{1}}$ generated by $-i \frac{d}{d \theta} \Lambda_{\mathbb{S}^{1}}$ and $\mathbf{C}^{\infty}\left(\mathbb{S}^{1}\right)$. In other words, $\mathscr{S} \mathscr{L}$ is the unique comparison algebra over $\mathbb{S}^{1}$. It therefore contains the compact ideal $\mathscr{K}_{\mathbb{S}^{1}}$ and all its commutators are compact (cf. [2], Chapters V and VI).

The following theorem was proven in [5] (Theorem 2.6). See also [7], Theorem 1.2.

THEOREM 1.4. With the above notation, we have:

$$
W \widehat{\mathscr{E}} W^{-1}=\mathscr{S} \mathscr{L} \bar{\otimes} \mathscr{K}_{\mathbb{Z}},
$$

where $\mathscr{K}_{\mathbb{Z}}$ denotes the set of compact operators on $L^{2}(\mathbb{Z})$. Furthermore, for $b \in \mathbf{C S}(\mathbb{R}), a \in \mathbf{C O}(\mathbb{R})$ and $j \in \mathbb{Z}$, we have:

$$
A^{\diamond}\left(e^{2 \pi i \varphi}\right):=Y_{\varphi} a(\varphi-M) Y_{-\varphi} \in \mathbf{C}\left(\mathbb{S}^{1}, \mathscr{K}_{\mathbb{Z}}\right)
$$

and

$$
\begin{aligned}
W\left(b(D) a T_{j}\right) W^{-1}=b\left(D_{\theta}\right) Y_{\varphi} a(\varphi-M) Y_{-\varphi-j}+K, & \\
K \in \mathscr{K}_{\mathbb{S}^{1} \times \mathbb{Z}} &
\end{aligned}
$$

Proposition 1.5. The map

$$
\begin{aligned}
\widehat{\mathscr{E}}_{\mathscr{P}} & \rightarrow \mathscr{S} \mathscr{L} \bar{\otimes} \mathscr{K}_{\mathbb{Z}} \bar{\otimes} \mathscr{K}_{\mathbb{B}}, \\
A & \mapsto W A W^{-1}
\end{aligned}
$$


is an onto *-isomorphism. For $A \in \widehat{\mathscr{C}}_{\mathscr{P}}$ of the form $A=b(D) K(\tau) T_{j}$, with $b \in \mathbf{C S}(\mathbb{R}), K(\tau) \in \mathbf{C O}\left(\mathbb{R}, \mathscr{H}_{\mathbb{B}}\right)$ and $j \in \mathbb{Z}$, we have:

(12) $W A W^{-1}=b\left(D_{\theta}\right) Y_{\varphi} K(\varphi-M) Y_{-\varphi-j}+K$, with $K \in \mathscr{K}_{\mathbb{S}^{1} \times \mathbb{Z} \times \mathbb{B}^{*}}$

For each $\varphi \in \mathbb{R}$ here, $K(\varphi-M)$ denotes the compact operator on $L^{2}(\mathbb{Z}) \bar{\otimes} L^{2}(\mathbb{B})$ defined by the sequence $K(\varphi-j) \in \mathscr{K}_{\mathbb{B}}, j \in \mathbb{Z}$. The first term of the right-hand side of (12) defines therefore a $\mathscr{K}_{\mathbb{Z} \times \mathbb{B} \text {-valued }}$ continuous function on $\mathbb{S}^{1}=\left\{e^{2 \pi i \varphi} ; \varphi \in \mathbb{R}\right\}$.

Proof. By Corollary 1.3 and (10),

$$
W \widehat{\mathscr{C}}_{\mathscr{P}} W^{-1}=\mathscr{S} \mathscr{L} \bar{\otimes} \mathscr{K}_{\mathbb{Z}} \bar{\otimes} \mathscr{K}_{\mathbb{B}}
$$

and, by (11), formula (12) holds for $K(\tau)$ of the form $a(\tau) \otimes \widetilde{K}$, $a \in \mathbf{C O}(\mathbb{R})$ and $\widetilde{K} \in \mathscr{K}_{\mathbb{B}}$. We can then find a sequence $K_{m}(\tau) \in$ $\mathbf{C O}\left(\mathbb{R}, \mathscr{K}_{\mathbb{B}}\right)$ such that $K_{m}(\tau) \rightarrow K(\tau)$, uniformly in $\tau \in \mathbb{R}$, and (12) is valid for each $K_{m}(\tau)$. Then

$$
Y_{\varphi} K_{m}(\varphi-M) Y_{-\varphi-j} \rightarrow Y_{\varphi} K(\varphi-M) Y_{-\varphi-j}
$$

in $\mathscr{K}_{\mathbb{Z} \times \mathbb{B}}$, uniformly in $e^{2 \pi i \varphi} \in \mathbb{S}^{1}$. Since the supremum-norm of a function on $\mathbb{S}^{1}$ taking values in $\mathscr{L}\left(L^{2}(\mathbb{Z}) \bar{\otimes} L^{2}(\mathbb{B})\right)$ equals the norm of the corresponding multiplication operator on $L^{2}\left(\mathbb{S}^{1}\right) \bar{\otimes} L^{2}(\mathbb{Z}) \bar{\otimes} L^{2}(\mathbb{B})$, the convergence above also holds in $\mathscr{L}\left(L^{2}\left(\mathbb{S}^{1}\right) \bar{\otimes} L^{2}(\mathbb{Z}) \bar{\otimes} L^{2}(\mathbb{B})\right)$.

Let $\mathbf{M}_{S L}$ denote the maximal-ideal space of $\mathscr{S} \mathscr{L} / \mathscr{K}_{\mathbb{S}^{1}}$ and let

$$
\sigma^{S L}: \mathscr{S} \mathscr{L} / \mathscr{K}_{\mathbb{S}^{1}} \rightarrow \mathbf{C}\left(\mathbf{M}_{S L}\right)
$$

denote the composition of the Gelfand map with the canonical projection. We then have (cf. [2], for example): $\mathbf{M}_{S L}=\mathbb{S}^{1} \times\{-1,+1\}$ and

$$
\sigma_{a}^{S L}(\cdot, \pm 1)=a(\cdot), \quad \text { for } a \in \mathbf{C}^{\infty}\left(\mathbb{S}^{1}\right)
$$

and

$$
\sigma_{b\left(D_{\theta}\right)}^{S L}(\cdot, \pm 1)=b( \pm \infty) \quad \text { for } b \in \mathbf{C S}(\mathbb{Z})
$$

Let $\mathbf{C}\left(\mathbf{M}_{S L}, \mathscr{K}_{\mathbb{Z} \times \mathbb{B}}\right)$ denote the $\mathscr{K}_{\mathbb{Z} \times \mathbb{B}}$-valued functions on $\mathbf{M}_{S L}$. Here $\mathscr{K}_{\mathbb{Z} \times \mathbb{B}}$ denotes the compact ideal of $L^{2}(\mathbb{Z}) \bar{\otimes} L^{2}(\mathbb{B}), \mathscr{K}_{\mathbb{Z} \times \mathbb{B}}=$ $\mathscr{K}_{\mathbb{Z}} \bar{\otimes} \mathscr{K}_{\mathbb{B}}$.

THEOREM 1.6. There exists an onto *-isomorphism

$$
\Psi: \frac{\mathscr{C}_{\mathscr{P}}}{\mathscr{K}(\mathscr{H})} \rightarrow \mathbf{C}\left(\mathbf{M}_{S L}, \mathscr{K}_{\mathbf{Z} \times \mathbb{B}}\right)
$$


such that if $\tilde{\gamma}$ denotes the composition of $\Psi$ with the canonical projection $\mathscr{E}_{\mathscr{P}} \rightarrow \mathscr{E}_{\mathscr{P}} / \mathscr{H}(\mathscr{H})$ and $A \in \mathscr{E}_{\mathscr{P}}$ is such that $B=F^{-1} A F$ is of the form $B=b(D) K(\tau) T_{j}$, where $b \in \mathbf{C S}(\mathbb{R}), K(\tau) \in \mathbf{C O}\left(\mathbb{R}, \mathscr{K}_{\mathbb{B}}\right)$ and $j \in \mathbb{Z}$, we then have:

$$
\tilde{\gamma}_{A}\left(e^{2 \pi i \varphi}, \pm 1\right)=b( \pm \infty) Y_{\varphi} K(\varphi-M) Y_{-\varphi-j}
$$

Proof. Let $\Psi$ be given by

$$
\frac{\mathscr{E}_{\mathscr{P}}}{\mathscr{K}(\mathscr{H})} \rightarrow \frac{\hat{\mathscr{E}}_{\mathscr{P}}}{\mathscr{K}(\mathscr{H})} \rightarrow \frac{\mathscr{\mathscr { L }} \bar{\otimes} \mathscr{K}_{\mathbb{Z}} \bar{\otimes} \mathscr{K}_{\mathbb{B}}}{\mathscr{K}_{\mathbb{S}^{1} \times \mathbb{Z} \times \mathbb{B}}} \rightarrow \mathbf{C}\left(M_{S L}, \mathscr{K}_{\mathbb{Z} \times \mathbb{B}}\right),
$$

where in the first step we take $A+\mathscr{K}(\mathscr{H}) \in \mathscr{C}_{\mathscr{P}} / \mathscr{K}(\mathscr{H})$ to $F^{-1} A F+$ $\mathscr{K}(\mathscr{H})$, next to

$$
W F^{-1} A F W^{-1}+\mathscr{H}_{\mathbb{S}^{1} \times \mathbb{Z} \times \mathbb{B}},
$$

and in the last step we use the onto $*$-isomorphism (see [1]):

$$
\frac{\mathscr{S} \mathscr{L} \bar{\otimes} \mathscr{K}_{\mathbb{Z}} \bar{\otimes} \mathscr{K}_{\mathbb{B}}}{\mathscr{K}_{\mathbb{S}^{1} \times \mathbb{Z} \times \mathbb{B}}} \rightarrow \mathbf{C}\left(M_{S L}, \mathscr{K}_{\mathbb{Z} \times \mathbb{B}}\right)
$$

$A \otimes K_{1} \otimes K_{2}+\mathscr{K}_{\mathbb{S}^{1} \times \mathbb{Z} \times \mathbb{B}} \mapsto \sigma_{A}^{S L}(\varphi, \pm 1) K_{1} \otimes K_{2}$.

Defined this way, $\Psi$ has the desired properties, by Proposition 1.5 and its proof.

2. Definition of two symbols on $\mathscr{C}_{\mathscr{P}}$. Our first task in this section is to give a precise description of the symbol space of $\mathscr{C}_{\mathscr{P}}$, i.e., the maximal-ideal space of the commutative $C^{*}$-algebra $\mathscr{C}_{\mathscr{P}} / \mathscr{E}_{\mathscr{P}}$. The symbol space of $\mathscr{C}$, the $C^{*}$-algebra generated by the operators listed in (1) except the periodic functions $e^{i j t}$, was described in [3]:

THEOREM 2.1 (Theorem 2.3 of [3]). The symbol space $\mathbf{M}$ of $\mathscr{C}$ can be identified with the bundle of unit spheres of the cotangent bundle of the compact manifold with boundary $[-\infty,+\infty] \times \mathbb{B}$, where $[-\infty,+\infty]$ denotes the compactification of $\mathbb{R}$ obtained by adding the points $-\infty$ and $+\infty$. The $\sigma$-symbols of the generators $A_{1}, A_{2}, A_{4}, A_{5}$ and $A_{6}$ are given below as functions of the local coordinates $(t, x ; \tau, \xi)$, where $(t, \tau) \in[-\infty,+\infty] \times \mathbb{R}^{*},(x, \xi) \in T^{*} \mathbb{B}$ and $\tau^{2}+h^{j k} \xi_{j} \xi_{k}=1:$

$$
\sigma_{A_{1}}=a(x), \quad \sigma_{A_{2}}=b(t), \quad \sigma_{A_{4}}=0, \quad \sigma_{A_{5}}=\tau, \quad \sigma_{A_{6}}=b^{j}(x) \xi_{j} .
$$


When periodic functions are adjoined to the algebra, the points over $|t|=\infty$ become circles. More precisely, we have:

THEOREM 2.2. The symbol space $\mathbf{M}_{P}$ of $\mathscr{C}_{\mathscr{P}}$ is homeomorphic to the closed subset of $\mathbf{M} \times \mathbb{S}^{1}$ described in local coordinates by

$$
\left\{\left((t, x ; \tau, \xi), e^{i \theta}\right) ;(t, x ; \tau, \xi) \in \mathbf{M}, \theta \in \mathbb{R} \text { and } \theta=t \text { if }|t|<\infty\right\} .
$$

Using this description of $\mathbf{M}_{P}$, the $\sigma$-symbols of the generators in (1) are respectively given by

$$
a(x), \quad b(t), \quad e^{i j \theta}, \quad 0, \quad \tau \text { and } b^{j}(x) \xi_{J} .
$$

Proof. Let $\mathbf{P}_{2 \pi}$ denote the closed algebra generated by $\left\{e^{i j t} ; j \in\right.$ $\mathbb{Z}\}$, i.e., the $2 \pi$-periodic continuous functions on $\mathbb{R}$. Its maximalideal space is $\mathbb{S}^{1}$, with $e^{i \theta} \in \mathbb{S}^{1}$ defining the multiplicative linear functional $f \rightarrow f(\theta)$.

With $\mathscr{E}$ denoting the commutator ideal of $\mathscr{C}$, the maximal-ideal space of $\mathscr{C} / \mathscr{E}$ is $\mathbf{M}$, as described in Theorem 2.1. By definition of the Gelfand map, a point $(t, x ; \tau, \xi)$ defines the multiplicative linear functional

$$
A+\mathscr{E} \rightarrow \sigma_{A}(t, x ; \tau, \xi) .
$$

The following maps are canonically defined:

$$
i_{1}: \frac{\mathscr{C}}{\mathscr{E}} \rightarrow \frac{\mathscr{C}_{\mathscr{P}}}{\mathscr{E}_{\mathscr{P}}}
$$

and

$$
i_{2}: \mathbf{P}_{2 \pi} \rightarrow \frac{\mathscr{C}_{\mathscr{P}}}{\mathscr{E}_{\mathscr{P}}}
$$

(It is obvious that $\mathscr{E} \subseteq \mathscr{E}_{\mathscr{P}}$ )

Let us denote by $l$ the product of the dual maps of $i_{1}$ and $i_{2}$, i.e.,

$$
\begin{aligned}
\imath: \mathbf{M}_{P} & \rightarrow \mathbf{M} \times \mathbb{S}^{1}, \\
w & \mapsto\left(w \circ i_{1},, w \circ i_{2}\right),
\end{aligned}
$$

where $w$ denotes a multiplicative linear functional on $\mathscr{C}_{\mathscr{P}} / \mathscr{E}_{\mathscr{P}}$.

As the images of $i_{1}$ and $i_{2}$ generate $\mathscr{C}_{\mathscr{P}} / \mathscr{E}_{\mathscr{P}}, l$ is an injective map, clearly continuous, which proves that $\mathbf{M}_{P}$ is homeomorphic to a compact subset of $\mathbf{M} \times \mathbb{S}^{1}$. Now we proceed to investigate which points of $\mathbf{M} \times \mathbb{S}^{1}$ belong to the image of $l$. This dual-map argument is essentially "Herman's Lemma" (cf. [4]).

As in the proof of Proposition 1.2, here again we use general results on comparison algebras. It follows from Theorem VII-1-5 of [2] that 
for every point of the cosphere-bundle of $\Omega,(t, x ; \tau, \xi) \in S^{*} \Omega$, there is a multiplicative linear functional on $\mathscr{C}_{\mathscr{P}} / \mathscr{C}_{\mathscr{P}}$ that takes any function $a$, belonging to the closed algebra generated by $A^{\sharp}$ in (6), to $a(x, t)$ and $D \Lambda$,

$$
D=\frac{1}{i} \frac{\partial}{\partial t}+\frac{1}{i} b^{j}(x) \frac{\partial}{\partial x^{j}}+q(x) \in \mathscr{D}^{\sharp},
$$

to $\tau+b^{j}(x) \xi_{j}$. This multiplicative linear functional must correspond to the point

$$
\left((t, x ; \tau, \xi), e^{i t}\right) \in \mathbf{M} \times \mathbb{S}^{1},
$$

with $|t|<\infty$.

Suppose now that $\left((t, x ; \tau, \xi), e^{i \theta}\right)$ is in the image of $l$ and that $|t|<\infty$. Let $\omega$ denote the corresponding multiplicative linear functional on $\mathscr{C}_{\mathscr{P}} / \mathscr{C}_{\mathscr{P}}$ and $\chi$ denote a function in $\mathbf{C}_{0}^{\infty}(\Omega)$ with $\chi(t)=1$. It is clear that $\chi(\cdot) e^{i(\cdot)}+\mathscr{E}_{\mathscr{P}}$ belongs to the image of $i_{1}$ and thus, by (15),

$$
\omega\left(\chi(\cdot) e^{i(\cdot)}+\mathscr{E}_{\mathscr{P}}\right)=e^{i t} .
$$

On the other hand, since $e^{i(\cdot)}+\mathscr{E}_{\mathscr{P}}$ belongs to the image of $i_{2}$, we get:

$$
\omega\left(\chi(\cdot) e^{i(\cdot)}+\mathscr{E}_{\mathscr{P}}\right)=\omega\left(\chi(\cdot)+\mathscr{E}_{\mathscr{P}}\right) \omega\left(e^{i(\cdot)}+\mathscr{E}_{\mathscr{P}}\right)=e^{i \theta} .
$$

We then obtain $e^{i \theta}=e^{i t}$.

For $t= \pm \infty$ and any $e^{i \theta} \in \mathbb{S}^{1}$, let us consider the sequence $t_{m}=$ $\theta \pm 2 \pi m$. Since $\mathbf{M}_{P}$ is closed and

$$
\left(\left(t_{m}, x ; \tau, \xi\right), e^{i t_{m}}\right) \rightarrow\left((t, x ; \tau, \xi), e^{i \theta}\right) \quad \text { as } m \rightarrow \infty,
$$

we conclude that $\left((t, x ; \tau, \xi), e^{i \theta}\right) \in \mathbf{M}_{P}$.

REMARK 2.3. We have just proved above that

$$
\mathbf{W}_{P}:=\left\{\left((t, x ; \tau, \xi), e^{i \theta}\right) \in \mathbf{M}_{P} ;|t|<\infty\right\}
$$

is dense in $\mathbf{M}_{P}$.

Next we define the $\gamma$-symbol.

The $C^{*}$-algebra $\mathscr{C}_{\mathscr{P}} / \mathscr{K}(\mathscr{H})$ has the closed two-sided ideal $\mathscr{E}_{\mathscr{P}} / \mathscr{K}(\mathscr{H})$, which was proven to be $*$-isomorphic to $\mathbf{C}\left(M_{S L}, \mathscr{K}_{\mathbb{Z} \times \mathbb{B}}\right)$ in Theorem 1.6. Every $A \in \mathscr{C}_{\mathscr{P}}$ determines a bounded operator of $\mathscr{L}\left(\mathscr{E}_{\mathscr{P}} / \mathscr{K}(\mathscr{H})\right)$ by $E+\mathscr{K}(\mathscr{H}) \rightarrow A E+\mathscr{K}(\mathscr{H})$, thus defining

$$
T: \mathscr{C}_{\mathscr{P}} \rightarrow \mathscr{L}\left(\mathscr{C}_{\mathscr{P}} / \mathscr{K}(\mathscr{H})\right) .
$$


Let us define

$$
\begin{aligned}
\gamma: \mathscr{C}_{\mathscr{P}} & \rightarrow \mathscr{L}\left(\mathbf{C}\left(\mathbf{M}_{S L}, \mathscr{K}_{\mathbb{Z} \times \mathbb{B}}\right)\right) \\
A & \mapsto \gamma_{A}=\Psi T_{A} \Psi^{-1},
\end{aligned}
$$

for $\Psi$ defined in Theorem 1.6.

For $E \in \mathscr{E}_{\mathscr{D}}, \gamma_{E}$ is the operator multiplication by $\tilde{\gamma}_{E} \in$ $\mathbf{C}\left(\mathbf{M}_{S L}, \mathscr{K}_{\mathbb{Z} \times \mathbb{B}}\right)$ (see Theorem 1.6). Let $\mathbf{C}\left(\mathbf{M}_{S L}, \mathscr{L}_{\mathbb{Z} \times \mathbb{B}}\right)$ denote the continuous functions on $\mathbf{M}_{S L}$ taking values in $\mathscr{L}_{\mathbb{Z} \times \mathbb{B}}:=$ $\mathscr{L}\left(L^{2}(\mathbb{Z} \times \mathbb{B})\right)$. Identifying functions in $\mathbf{C}\left(\mathbf{M}_{S L}, \mathscr{L}_{\mathbb{Z} \times \mathbb{B}}\right)$ with the corresponding multiplication operator of $\mathscr{L}\left(\mathbf{C}\left(\mathscr{M}_{S L}, \mathscr{K}_{\mathbb{Z} \times \mathbb{B}}\right)\right)$, we can say then that $\gamma$ is an extension of $\tilde{\gamma}$.

Proposition 2.4. There exists a *-homomorphism

$$
\gamma: \mathscr{C}_{\mathscr{P}} \rightarrow \mathbf{C}\left(\mathbf{M}_{S L}, \mathscr{L}_{\mathbb{Z} \times \mathbb{B}}\right),
$$

where

$$
\mathbf{M}_{S L}=\left\{e^{2 \pi i \varphi} ; \varphi \in \mathbb{R}\right\} \times\{+1,-1\},
$$

given on the generators (1), according to notation established in $\S 1$ and in Theorem 1.6, by:

$$
\begin{gathered}
\gamma_{A_{1}}=a(x) ; \quad \gamma_{A_{2}}=b( \pm \infty) \\
\gamma_{A_{3}}=Y_{-j} ; \quad \gamma_{A_{4}}=Y_{\varphi} \tilde{\Lambda}(\varphi-M) Y_{-\varphi} ; \\
\gamma_{A_{5}}=Y_{\varphi} K(\varphi-M) Y_{-\varphi}, \quad \text { where } K(\tau)=-\tau \widetilde{\Lambda}(\tau), \tau \in \mathbb{R} \text { and } \\
\gamma_{A_{6}}=Y_{\varphi} L(\varphi-M) Y_{-\varphi}, \quad \text { where } L(\tau)=D_{x} \tilde{\Lambda}(\tau), \tau \in \mathbb{R} .
\end{gathered}
$$

Furthermore, $\gamma$ restricted to the $C^{*}$-algebra $C_{\mathscr{D}}^{\diamond}$, generated by the operators in (1) except $b \in \mathbf{C S}(\mathbb{R})$, is an isometry.

Proof. Let us calculate $\gamma$, defined in (16), for the generators $A_{1}, \ldots, A_{6}$ of (1). By Proposition 1.2, it is enough to calculate the result of a left multiplication by $A_{p}, p=1, \ldots, 6$, on operators $E \in \mathscr{E}_{\mathscr{P}}$ such that $F^{-1} E F$ are of the form $c(D) K(\tau) T_{l}, c \in \mathbf{C S}(\mathbb{R})$, $K \in \mathbf{C O}\left(\mathbb{R}, \mathscr{K}_{\mathbb{B}}\right)$ and $l \in \mathbb{Z}$. For such an $E$, we get $F^{-1}\left(A_{p} E\right) F$, $p=1, \ldots, 6$, equal to, modulo compact operators,

$$
c(D) a(x) K(\tau) T_{l}, \quad(c b)(D) K(\tau) T_{l}, \quad c(D) K(\tau+j) T_{j+l},
$$

$$
c(D) \widetilde{\Lambda}(\tau) K(\tau) T_{l}, \quad-c(D) \tau \widetilde{\Lambda}(\tau) K(\tau) T_{l} \quad \text { and } \quad c(D) D_{x} \widetilde{\Lambda}(\tau) K(\tau) T_{l}
$$

respectively. Here we have used (3) and

$$
\left[c(D), B_{k}\right] \in \mathscr{K}(\mathscr{H}), \quad k=4,5,6
$$


(cf. [3], Proposition 1.2). By Theorem 1.6, we get:

$$
\begin{aligned}
\gamma_{A_{1} E}(\varphi, \pm 1) & =c( \pm \infty) Y_{\varphi} \widetilde{K}(\varphi-M) Y_{-\varphi-1} \\
& =a(x) \gamma_{E}(\varphi, \pm 1) \quad(\widetilde{K}=a K), \\
\gamma_{A_{2} E}(\varphi, \pm 1) & =(c b)( \pm \infty) Y_{\varphi} K(\varphi-M) Y_{-\varphi-1}=b( \pm \infty) \gamma_{E}(\varphi, \pm \infty), \\
\gamma_{A_{3} E}(\varphi, \pm 1) & =c( \pm \infty) Y_{\varphi} K(\varphi+j-M) Y_{-\varphi-j-l}=Y_{j} \gamma_{E}(\varphi, \pm 1), \\
\gamma_{A_{4} E}(\varphi, \pm 1) & =c( \pm \infty) Y_{\varphi}(\widetilde{\Lambda} K)(\varphi-M) Y_{-\varphi-l} \\
& =Y_{\varphi} \widetilde{\Lambda}(\varphi-M) Y_{-\varphi} \gamma_{E}(\varphi, \pm 1)
\end{aligned}
$$

and analogously for $p=5$ and 6. This proves formulas (17).

For any $A \in \mathscr{C}_{\mathscr{P}}$ such that $F^{-1} A F=J(\tau) \in \mathbf{C O}\left(\mathbb{R}, \mathscr{K}_{\mathbb{B}}\right)$, it is also clear, using $(5)$, that

$$
\gamma_{A}(\varphi, \pm 1)=Y_{\varphi} J(\varphi-M) Y_{-\varphi} .
$$

Hence, by (4), $\gamma_{A_{6}^{*}}$ also belongs to $\mathbf{C}\left(\mathbf{M}_{S L}, \mathscr{L}_{\mathbb{Z} \times \mathbb{B}}\right)$.

The norm of the operator of $\mathscr{L}\left(\mathbf{C}\left(\mathbf{M}_{S L}, \mathscr{K}_{\mathbb{Z} \times \mathbb{B}}\right)\right)$ given by multiplication by a function in $\mathbf{C}\left(\mathbf{M}_{S L}, \mathscr{L}_{\mathbb{Z} \times \mathbb{B}}\right)$ is equal to the sup-norm of this function. In other words, the $C^{*}$-algebra $\mathbf{C}\left(\mathbf{M}_{S L}, \mathscr{L}_{\mathbb{Z} \times \mathbb{B}}\right)$ is isometrically imbedded in $\mathscr{L}\left(\mathbf{C}\left(\mathbf{M}_{S L}, \mathscr{K}_{\mathbb{Z} \times \mathbb{B}}\right)\right)$. As the image of a dense subalgebra of $\mathscr{C}_{\mathbb{P}}$ is contained in $\mathbf{C}\left(\mathbf{M}_{S L}, \mathscr{L}_{\mathbf{Z} \times \mathbb{B}}\right)$, we conclude that $\gamma$ maps $\mathscr{C}_{\mathscr{P}}$ into $\mathbf{C}\left(\mathbf{M}_{S L}, \mathscr{L}_{\mathbb{Z} \times \mathbb{B}}\right)$.

Using the identification

$$
L^{2}\left(\mathbb{S}^{1}\right) \bar{\otimes} L^{2}(\mathbb{Z}) \bar{\otimes} L^{2}(\mathbb{B})=L^{2}\left(\mathbb{S}^{1}, L^{2}(\mathbb{Z} \times \mathbb{B})\right),
$$

it can be straightforwardly verified that, for $A(\tau) \in \mathbf{C B}\left(\mathbb{R}, \mathscr{L}_{\mathbb{B}}\right)$, $W A(\tau) W^{-1} \in \mathbf{C}\left(\mathbb{S}^{1}, \mathscr{L}_{\mathbb{Z} \times \mathbb{B}}\right)$ and it is given by $Y_{\varphi} A(\varphi-M) Y_{-\varphi}$. This means that for $k=1,4,5,6$, we have

$$
\gamma_{A_{k}}=W F^{-1} A_{k} F W^{-1} \text { and } \gamma_{A_{k}^{*}}=W F^{-1} A_{k}^{*} F W^{-1} .
$$

It is also clear that $W T_{j} W^{-1}=Y_{-j}$ and, hence,

$$
\gamma_{A}=W F^{-1} A\left(W F^{-1}\right)^{-1}, \quad \text { for } A \in \mathscr{C}_{\mathscr{P}}^{\diamond},
$$

proving that

$$
\left\|\gamma_{A}\right\|_{\mathbf{C}\left(\mathbf{M}_{S L}, \mathscr{L}_{\mathbb{Z} \times \mathbb{B}}\right)}=\|A\|_{\mathscr{L}(\mathscr{L})} \quad \text { and } \quad \gamma_{A^{*}}=(\gamma A)^{*} \quad \text { for } A \in \mathscr{C}_{\mathscr{P}}^{\diamond} \text {. }
$$

This finishes the proof, since it is obvious that $\gamma_{A_{2}^{*}}=\left(\gamma_{A_{2}}\right)^{*}$. 
The $\sigma$-symbol and the $\gamma$-symbol, defined in Theorem 2.2 and Proposition 2.4 respectively, are related by:

Proposition 2.5. For every $A \in \mathscr{C}_{\mathscr{P}},\left\|\left.\sigma_{A}\right|_{\mathbf{M}_{P} \backslash \mathbf{w}_{P}}\right\| \leq\left\|\gamma_{A}\right\|$, i.e., $\sup \left\{\left|\sigma_{A}\left((t, x ; \tau, \xi), e^{i \theta}\right)\right| ;|t|=\infty\right\} \leq \sup \left\{\left\|\gamma_{A}(m)\right\|_{\mathscr{L}_{\mathrm{Z} \times \mathbb{B}}} ; m \in \mathbf{M}_{S L}\right\}$

Proof. Since the commutators of $A_{2}$ with the other generators in (1) and their adjoints are compact (cf. [3], Proposition 1.2), the set of operators of the form

$$
\begin{aligned}
& A=\sum_{j=1}^{N} b_{j}(t) A_{j}+K, \\
& \qquad b_{j} \in \mathbf{C S}(\mathbb{R}), A_{j} \in \mathscr{C}_{\mathscr{P}}^{\diamond}, K \in \mathscr{K}(\mathscr{H}), N \in \mathbb{N},
\end{aligned}
$$

is dense in $\mathscr{C}_{\mathscr{P}}$. As $\sigma_{K}=0$ and $\gamma_{K}=0$ for $K \in \mathscr{K}(\mathscr{H})$, it suffices to assume $A$ of the form (18) with $K=0$.

For such an $A$, Theorem 2.2 implies:

$$
\sigma_{A}\left((t, x ; \tau, \xi), e^{i \theta}\right)=\sum_{j=1}^{N} b_{j}(t) \sigma_{A_{j}}\left((t, x ; \tau, \xi), e^{i \theta}\right) .
$$

Letting $A^{ \pm}$denote the operators $\sum_{j=1}^{N} b_{j}( \pm \infty) A_{j}$, it is clear then that

$$
\begin{aligned}
& \sigma_{A}\left((+\infty, x ; \tau, \xi), e^{i \theta}\right)=\sigma_{A^{+}}\left(( \pm \infty, x ; \tau, \xi), e^{i \theta}\right) \quad \text { and } \\
& \sigma_{A}\left((-\infty, x ; \tau, \xi), e^{i \theta}\right)=\sigma_{A_{-}}\left(( \pm \infty, x ; \tau, \xi), e^{i \theta}\right)
\end{aligned}
$$

hence:

$$
\left\|\left.\sigma_{A}\right|_{\mathbf{M}_{P} \backslash \mathbf{W}_{P}}\right\| \leq \max \left\{\left\|\sigma_{A^{+}}\right\|,\left\|\sigma_{A^{-}}\right\|\right\} .
$$

The map $\sigma: \mathscr{C}_{\mathscr{P}} \rightarrow \mathbf{C}\left(\mathbf{M}_{P}\right)$ was defined as the composition of the Gelfand map (an isometry) with the canonical projection $\mathscr{C}_{\mathscr{P}} \rightarrow$ $\mathscr{C}_{\mathscr{P}} / \mathscr{K}(\mathscr{H})$. It then follows that

$$
\left\|\sigma_{A^{ \pm}}\right\| \leq\left\|A^{ \pm}\right\| .
$$

As $A^{ \pm} \in \mathscr{C}_{\mathscr{P}}^{\diamond}$, where $\gamma$ is an isometry,

$$
\left\|\sigma_{A^{ \pm}}\right\|_{\mathbf{C}\left(\mathbf{M}_{P}\right)} \leq\left\|\gamma_{A^{ \pm}}\right\|_{\mathbf{C}\left(\mathbf{M}_{S L}, \mathscr{L}_{\mathbb{Z} \times \mathbb{B}}\right)} .
$$

By Proposition 2.4,

$$
\gamma_{A}(\varphi,+1)=\sum_{j=1}^{N} b_{j}(+\infty) \gamma_{A_{j}}(\varphi,+1)=\gamma_{A^{+}}(\varphi,+1)
$$


and

$$
\gamma_{A}(\varphi,-1)=\gamma_{A^{-}}(\varphi,-1) .
$$

Furthermore, for any $A \in \mathscr{C}_{\mathscr{P}}^{\diamond}$, it is clear from (17) that $\gamma_{A}(\varphi,+1)=$ $\gamma_{A}(\varphi,-1)$ and, therefore,

$$
\left\|\gamma_{A}\right\|=\max \left\{\left\|\gamma_{A^{+}}\right\|,\left\|\gamma_{A^{-}}\right\|\right\}
$$

We are finished by (19), (20) and (21).

If $\gamma_{A}=0$, then, $\left.\sigma_{A}\right|_{\mathbf{M}_{P} \backslash \mathbf{w}_{P}}=0$. The converse is also true:

Proposition 2.6. An operator $A \in \mathscr{C}_{\mathscr{P}}$ belongs to the kernel of $\gamma$ if and only if $\sigma_{A}$ vanishes on $\mathbf{M}_{P} \backslash \mathbf{W}_{P}$. Furthermore, we have:

$$
\operatorname{ker} \gamma \cap \operatorname{ker} \sigma=\mathscr{K}(\mathscr{H}) \text {. }
$$

Proof. Let $\mathscr{T}_{0}$ denote the $C^{*}$-algebra generated by multiplications by functions in $C_{0}^{\infty}(\Omega)$ and by the operators of the form $D \Lambda$, where $D$ is a first order linear differential operator on $\Omega$ with smooth coefficients of compact support. Given $A_{0}$, one of these generators just described, we can find $\chi \in \mathbf{C}_{0}^{\infty}(\mathbb{R})$ such that $\chi A_{0}=A_{0}$ and then $\gamma_{A_{0}}=\gamma_{\chi} \gamma_{A_{0}}=0$, by Proposition 2.4. So, we have $\mathscr{T}_{0} \subseteq \operatorname{ker} \gamma$.

Using the nomenclature of [2], $\mathscr{T}_{0}$ is the minimal comparison algebra associated with the triple $\{\Omega, d S, H\}$. It can be easily concluded from [2], Lemma VII-1-2, that $A \in \mathscr{C}_{\mathscr{P}}$ belongs to $\mathscr{T}_{0}$ if and only if $\sigma_{A}$ vanishes on $\mathbf{M}_{P} \backslash \mathbf{W}_{P}$, proving that $\mathscr{T}_{0} \subseteq \operatorname{ker} \gamma$, by Proposition 2.5.

Since $\operatorname{ker} \sigma=\mathscr{E}_{\mathscr{P}}$ and $\operatorname{ker} \gamma=\mathscr{T}_{0}$, the equality in (22) follows from [2], Theorem VII-1-3.

3. A Fredholm criterion and an application to differential operators. We will now give a necessary and sufficient criterion for an $N \times N$-matrix whose entries are operators in $\mathscr{C}_{\mathscr{P}}$, regarded as a bounded operator on $L^{2}\left(\Omega, \mathbb{C}^{N}\right), N \geq 1$, to be Fredholm. Let us denote $L^{2}\left(\Omega, \mathbb{C}^{N}\right)$ by $\mathscr{H}^{N}$ and by $\mathscr{C}_{\mathscr{D}}^{N}$ the $C^{*}$-subalgebra of $\mathscr{L}\left(\mathscr{H}^{N}\right)$

$$
\mathscr{C}_{\mathscr{P}}^{N}:=\left\{\left(\left(A_{j k}\right)\right) ; A_{j k} \in \mathscr{C}_{\mathscr{P}}, 1 \leq j, k \leq N\right\} .
$$

It is easy to see that the compact ideal of $\mathscr{L}\left(\mathscr{H}^{N}\right)$ coincides with the matrices with entries in $\mathscr{K}(\mathscr{H})$, i.e.,

$$
\mathscr{K}\left(\mathscr{H}^{N}\right)=\mathscr{K}^{N}:=\left\{\left(\left(K_{j k}\right)\right) ; K_{j k} \in \mathscr{K}(\mathscr{H}), 1 \leq j, k \leq N\right\} .
$$

Let us define two symbols on $\mathscr{C}_{\mathscr{P}}^{N}$ :

$$
\sigma_{A}^{N}=\left(\left(\sigma_{A_{j k}}\right)\right)_{1 \leq j, k \leq N} \text { and } \gamma_{A}^{N}=\left(\left(\gamma_{A_{j k}}\right)\right)_{1 \leq j, k \leq N},
$$


where $A=\left(\left(A_{j k}\right)\right)_{1 \leq j, k \leq N} \in \mathscr{C}_{\mathscr{P}}^{N}$. The following proposition follows immediately from the definitions above and Proposition 2.6.

Proposition 3.1. The $\gamma^{N}$-symbol of an operator $A \in \mathscr{C}_{\mathscr{P}}^{N}$ is identically zero if and only if its $\sigma^{N}$-symbol vanishes on $\mathbf{M}_{P} \backslash \mathbf{W}_{P}$. Furthermore, we have:

$$
\operatorname{ker} \sigma^{N} \cap \operatorname{ker} \gamma^{N}=\mathscr{K}^{N}
$$

TheOREM 3.2. For an operator $A=\left(\left(A_{j k}\right)\right)_{1 \leq j, k \leq N} \in \mathscr{C}_{\mathscr{P}}^{N}$ to be Fredholm, it is necessary and sufficient that:

(i) $\sigma_{A}^{N}$ be invertible, i.e., the $N \times N$-matrix $\left(\left(\sigma_{A_{j k}}(m)\right)\right)$ be invertible for all $m \in \mathbf{M}_{P}$, and

(ii) $\gamma_{A}^{N}$ be invertible, i.e., the $N \times N$-matrix, with entries in $\mathbf{C}\left(\mathbf{M}_{S L}, \mathscr{L}_{\mathbb{Z} \times \mathbb{B}}\right),\left(\left(\gamma_{A_{j k}}(m)\right)\right)$ be invertible for all $m \in \mathbf{M}_{S L}$.

Proof. Suppose that $A$ is Fredholm and let $B$ be such that $1-A B$ and $1-B A$ are compact. We have $B \in \mathscr{C}_{\mathscr{P}}^{N}$, since $\mathscr{C}_{\mathscr{P}}^{N} / \mathscr{K}^{N}$ is a $C^{*}$-subalgebra of $\mathscr{L}\left(\mathscr{H}^{N}\right) / \mathscr{K}^{N}$. We then get

$$
\sigma_{1-A B}^{N}=\sigma_{1-B A}^{N}=0 \text { and } \gamma_{1-A B}^{N}=\gamma_{1-B A}^{N}=0
$$

and, hence,

$$
1=\sigma_{A}^{N} \sigma_{B}^{N}=\sigma_{B}^{N} \sigma_{A}^{N} \text { and } 1=\gamma_{A}^{N} \gamma_{B}^{N}=\gamma_{B}^{N} \gamma_{A}^{N} .
$$

Conversely, suppose that (i) and (ii) above are satisfied. Since $\gamma^{N}: \mathscr{C}_{\mathscr{D}}^{N} \rightarrow \mathbf{C}\left(\mathbf{M}_{S L}, N \times N\right.$-matrices with entries in $\left.\mathscr{L}\left(L^{2}(\mathbb{Z}) \bar{\otimes} L^{2}(\mathbb{B})\right)\right)$ is a $*$-homomorphism (by Proposition 2.4), its range is a $C^{*}$-algebra. There must be then a $B \in \mathscr{C}_{\mathscr{P}}^{N}$ such that $\gamma_{B}^{N}=\left(\gamma_{A}^{N}\right)^{-1}$. Since $1-A B \in \operatorname{ker} \gamma^{N}, 1-\sigma_{A}^{N} \sigma_{B}^{N}$ vanishes on $\mathbf{M}_{P} \backslash \mathbf{W}_{P}$, by Proposition 3.1. As the map $\sigma$ is surjective, so is $\sigma^{N}$. An operator $Q \in \mathscr{C}_{\mathscr{P}}^{N}$ can therefore be found such that its symbol $\sigma_{Q}^{N}$ equals the continuous function vanishing on $M_{P} \backslash \mathbf{W}_{P}$

$$
\left(\sigma_{A}^{N}\right)^{-1}-\sigma_{B}^{N}
$$

By Proposition 3.1 again, $Q \in \operatorname{ker} \gamma^{N}$ and, then,

$$
\gamma_{1-A(B+Q)}^{N}=\gamma_{1-(B+Q) A}^{N}=0 \text {. }
$$

Since we also have

$$
\sigma_{1-A(B+Q)}^{N}=1-\sigma_{A}^{N} \sigma_{B}^{N}-\sigma_{A}^{N} \sigma_{Q}^{N}=0=\sigma_{1-(B+Q) A}^{N},
$$

the operator $B+Q$ is an inverse for $A$, modulo a compact operator, by equation (23). 
In order to apply this result to differential operators, it is convenient to conjugate the $\gamma$-symbol with the discrete Fourier transform. We define:

$$
\begin{aligned}
\Gamma: \mathscr{C}_{\mathscr{P}} & \rightarrow \mathbf{C}\left(\mathbf{M}_{S L}, \mathscr{L}_{\mathbb{S}^{1} \times \mathbb{B}}\right) \\
A & \mapsto \Gamma_{A}(m)=F_{d}^{-1} \gamma_{A}(m) F_{d}, \quad m \in \mathbf{M}_{S L},
\end{aligned}
$$

where $F_{d}: L^{2}\left(\mathbb{S}^{1}\right) \rightarrow L^{2}(\mathbb{Z}), \mathbb{S}^{1}=\left\{e^{i \theta} ; \theta \in \mathbb{R}\right\}$, was defined in (7), and, as usual, $F_{d}$ also denotes $F_{d} \otimes I_{\mathbb{B}}$.

Next we calculate $\Gamma_{A}$ for the generators of $\mathscr{C}_{\mathscr{P}}$. It is obvious that, for $a \in \mathbb{C}^{\infty}(\mathbb{B})$,

$$
\Gamma_{a}(\varphi, \pm 1)=a, \quad\left(e^{2 \pi i \varphi}, \pm 1\right) \in \mathbf{M}_{S L},
$$

and, for $b \in \mathbf{C S}(\mathbb{R})$,

$$
\Gamma_{b}(\varphi, \pm 1)=b( \pm \infty), \quad \text { independent of } \varphi .
$$

For $j \in \mathbb{Z}, F_{d}^{-1} Y_{-j} F_{d}$ equals the operator multiplication by $e^{i j \theta}$ on $\mathbb{S}^{1}=\left\{e^{l \theta}, \theta \in \mathbb{R}\right\}$, and then, by (24) and (17),

$$
\Gamma_{e^{+i \jmath t}}(\varphi, \pm 1)=e^{i j \theta}, \quad \text { for all }\left(e^{2 \pi l \varphi}, \pm 1\right) \in \mathbf{M}_{S L} .
$$

Let $a \in \mathbf{C}(\Omega)$ be of the form

$$
a(t, x)=a_{+}(t, x) \chi_{+}(t, x)+a_{-}(t, x) \chi_{-}(t, x)+a_{0}(t, x),
$$

where $a_{ \pm}$are continuous and $2 \pi$-periodic in $t, a_{0} \in \mathbf{C O}(\Omega)$ and $\chi_{ \pm} \in \mathbf{C S}(\mathbb{R})$ satisfy $\chi_{ \pm}( \pm \infty)=1, \chi_{+}+\chi_{-}=1$. By the continuity of $\Gamma,(25),(26)$ and $(27)$, it follows that

$$
\Gamma_{a}(\varphi, \pm 1)=a_{ \pm}(\theta, x), \quad \text { for }\left(e^{2 \pi i \varphi}, \pm 1\right) \in \mathbf{M}_{S L} .
$$

Note that (28) gives $\Gamma_{A_{1}}, \Gamma_{A_{2}}$ and $\Gamma_{A_{3}}$, for $A_{p}$ as defined on page 283.

Now we calculate $F_{d}^{-1} K(\varphi-M) F_{d}$, for $\varphi \in \mathbb{R}$ and $K(\tau)=\widetilde{\Lambda}(\tau)$, $-\tau \widetilde{\Lambda}(\tau)$ or $D_{x} \widetilde{\Lambda}(\tau)$, which is needed for obtaining $\Gamma_{A_{p}}, p=4,5,6$. Let us use again that $-\Delta_{\mathbb{B}}$ has an orthonormal basis of eigenfunctions $w_{m}, \quad m \in \mathbb{N}$, with eigenvalues $0 \leq \lambda_{1} \leq \lambda_{2} \leq \cdots, \lambda_{m} \rightarrow \infty$ as $m \rightarrow \infty$, and define the unitary map

$$
\begin{aligned}
U: L^{2}(\mathbb{B}) & \rightarrow L^{2}(\mathbb{N}), \\
u & \mapsto\left(w_{m}, u\right)_{m \in \mathbb{N}} .
\end{aligned}
$$

By the spectral theorem, the conjugate $U\left(1+(\varphi-j)^{2}-\Delta_{\mathbb{B}}\right)^{-1 / 2} U^{-1}$ equals the operator multiplication by $\left(1+(\varphi-j)^{2}+\lambda_{m}\right)^{-1 / 2}$ on $L^{2}(\mathbb{N})$, 
for each $j \in \mathbb{Z}, \varphi \in \mathbb{R}$. The operator $\tilde{\Lambda}(\varphi-M) \in \mathscr{L}_{\mathbb{Z} \times \mathbb{B}}$ acts on

$$
\mathbf{u}=\left(u_{j}\right)_{j \in \mathbb{Z}} \in L^{2}\left(\mathbb{Z} ; L^{2}(\mathbb{B})\right)
$$

by

$$
\widetilde{\Lambda}(\varphi-M) \mathbf{u}=\left(\left(1+(\varphi-j)^{2}+\Delta_{\mathbb{B}}\right)^{-1 / 2} u_{j}\right)_{j \in \mathbb{Z}}
$$

and, thus,

$$
\left(I_{\mathbb{Z}} \otimes U\right) \tilde{\Lambda}(\varphi-M)\left(I_{\mathbb{Z}} \otimes U\right)^{-1}=\left(1+(\varphi-j)^{2}+\lambda_{m}\right)^{-1 / 2},
$$

where, by $\left(1+(\varphi-j)^{2}+\lambda_{m}\right)^{-1 / 2}$, we now mean the corresponding multiplication operator on $L^{2}(\mathbb{Z}) \bar{\otimes} L^{2}(\mathbb{N})$.

Let us adopt the notation:

$$
1+\left(\varphi-D_{\theta}\right)^{2}-\Delta_{\mathbb{B}}:=\left(F_{d} \otimes U\right)^{-1}\left(1+(\varphi-j)^{2}+\lambda_{m}\right)\left(F_{d} \otimes U\right)
$$

It is easy to see that $1+\left(\varphi-D_{\theta}\right)^{2}-\Delta_{\mathbb{B}}$ is the unique self-adjoint realization of the differential expression $1+\left(\varphi+i \frac{\partial}{\partial \theta}\right)^{2}-\Delta_{\mathbb{B}}$ on $\mathbb{S}^{1} \times \mathbb{B}$ (see Lemma 3.3). By (30) and (31) then, we obtain:

$$
\left(F_{d} \otimes I_{\mathbb{B}}\right)^{-1} \tilde{\Lambda}(\varphi-M)\left(F_{d} \otimes I_{\mathbb{B}}\right)=\left(1+\left(\varphi-D_{\theta}\right)^{2}-\Delta_{\mathbb{B}}\right)^{-1 / 2},
$$

for every $\varphi \in \mathbb{R}$. Using that $Y_{\varphi}=F_{d}^{-1} e^{-i \varphi \theta} F_{d}, \varphi \in \mathbb{R}$ and (17), it follows that:

$$
\begin{aligned}
\Gamma_{\Lambda}(\varphi, \pm 1)=e^{-i \varphi \theta}\left(1+\left(D_{\theta}-\varphi\right)^{2}-\Delta_{\mathbb{B}}\right)^{-1 / 2} e^{i \varphi \theta} & \\
& \left(e^{2 \pi i \varphi}, \pm 1\right) \in \mathbf{M}_{S L} .
\end{aligned}
$$

Since, for each $j \in \mathbb{Z}$ and each $\varphi \in \mathbb{R}$,

$$
U(\varphi-j)\left(1+(\varphi-j)^{2}-\Delta_{\mathbb{B}}\right)^{-1 / 2} U^{-1}
$$

equals the operator multiplication by

$$
(\varphi-j)\left(1+(\varphi-j)^{2}+\lambda_{m}\right)^{-1 / 2}
$$

on $L^{2}(\mathbb{N})$, we obtain, in a way analogous to how (33) was obtained:

$$
\begin{array}{r}
\Gamma_{A_{4}}(\varphi, \pm 1)=e^{-i \varphi \theta}\left(D_{\theta}-\varphi\right)\left(1+\left(D_{\theta}-\varphi\right)^{2}-\Delta_{\mathbb{B}}\right)^{-1 / 2} e^{i \varphi \theta}, \\
\left(e^{2 \pi i \varphi}, \pm 1\right) \in \mathbf{M}_{S L} .
\end{array}
$$

Here we have assumed the notation:

$$
\begin{aligned}
& \left(\varphi-D_{\theta}\right)\left(1+\left(\varphi-D_{\theta}\right)^{2}-\Delta_{\mathbb{B}}\right)^{-1 / 2} \\
& \quad:=\left(F_{d} \otimes U\right)^{-1}(\varphi-j)\left(1+(\varphi-j)^{2}+\lambda_{m}\right)^{-1 / 2}\left(F_{d} \otimes U\right) .
\end{aligned}
$$

For the last type of generator, we need the following lemma. 
LEMMA 3.3. The subspace

$$
\left\{u \in L^{2}\left(\mathbb{S}^{1} \times \mathbb{B}\right) ;\left(1+\left(\varphi-D_{\theta}\right)^{2}-\Delta_{\mathbb{B}}\right)^{-1 / 2} u \in \mathbb{C}^{\infty}\left(\mathbb{S}^{1} \times \mathbb{B}\right)\right\}
$$

is dense in $L^{2}\left(\mathbb{S}^{1} \times \mathbb{B}\right)$, for every $\varphi \in \mathbb{R}$.

Proof. The statement is true for $\varphi=0$, since

$$
1+D_{\theta}^{2}-\Delta_{\mathbb{B}}=1-\Delta_{\mathbb{S}^{1} \times \mathbb{B}}
$$

is essentially self-adjoint on $\mathbf{C}^{\infty}\left(\mathbb{S}^{1} \times \mathbb{B}\right)$, by [2], Theorem IV-1-8, for example. For $\varphi \in \mathbb{R}$,

$$
\left(1+\left(\varphi-D_{\theta}\right)^{2}-\Delta_{\mathbb{B}}\right)^{-1 / 2}\left(1+D_{\theta}^{2}-\Delta_{\mathbb{B}}\right)^{1 / 2}
$$

is a Banach-space isomorphism, since it is unitarily equivalent to the multiplication by the function on $\mathbb{Z} \times \mathbb{N}$

$$
\left(1+(\varphi-j)^{2}+\lambda_{m}\right)^{-1 / 2}\left(1+j^{2}+\lambda_{m}\right)^{-1 / 2},
$$

which is bounded and bounded away from zero.

For every $v \in \mathbf{C}^{\infty}\left(\mathbb{S}^{1} \times \mathbb{B}\right)$, it is clear that

$$
D_{x} F_{d} v=F_{d} D_{x} v \text {, }
$$

where, on the right-hand side, $D_{x}$ is regarded as a differential expression on $\mathbb{S}^{1} \times \mathbb{B}$ and, on the left-hand side, $D_{x}$ acts, as a differential operator on $\mathbb{B}$, on each component $w_{j} \in \mathbf{C}^{\infty}(\mathbb{B})$ of

$$
w=\left(w_{j}\right)_{j \in \mathbb{Z}}=F_{d} v \in L^{2}\left(\mathbb{Z} ; L^{2}(\mathbb{B})\right) .
$$

By Lemma 3.3, it therefore follows that

$$
\begin{aligned}
& F_{d}\left[D_{x}\left(1+\left(\varphi-D_{\theta}\right)^{2}-\Delta_{\mathbb{B}}\right)^{-1 / 2}\right] F_{d}^{-1} \\
& \quad=D_{x}\left[F_{d}\left(1+\left(\varphi-D_{\theta}\right)^{2}-\Delta_{\mathbb{B}}\right)^{-1 / 2} F_{d}^{-1}\right] .
\end{aligned}
$$

The right-hand side of (35) equals $D_{z} \widetilde{\Lambda}(\varphi-M)$, by (32). We have, hence:

$$
\Gamma_{A_{6}}(\varphi, \pm 1)=e^{-i \varphi \theta}\left[D_{x}\left(1+\left(\varphi-D_{\theta}\right)^{2}-\Delta_{\mathbb{B}}\right)^{-1 / 2}\right] e^{i \varphi \theta} .
$$

Equations (29), (31), (32), (33), (34) and (36) prove:

Proposition 3.4. The map $\Gamma$ defined in (24) is given on the generators of $\mathscr{C}_{\mathscr{P}}$ (with $m=\left(e^{2 \pi i \varphi}, \pm 1\right) \in \mathbf{M}_{S L}$ and $\Gamma_{A}(\varphi, \pm 1) \in \mathscr{L}_{\mathbb{S}^{1} \times \mathbb{B}}$, $\left.\mathbb{S}^{1}=\left\{e^{i \theta} ; \theta \in \mathbb{R}\right\}\right) b y:$

$$
\begin{aligned}
& \Gamma_{a}(\varphi, \pm 1)=a_{ \pm}(\theta, x), \text { for a as in }(28) \\
& \Gamma_{\Lambda}(\varphi, \pm 1)=e^{-i \varphi \theta}\left(1+\left(D_{\theta}-\varphi\right)^{2}-\Delta_{\mathbb{B}}\right)^{-1 / 2} e^{i \varphi \theta} \\
& \Gamma_{-i \frac{\partial}{\partial t} \Lambda}(\varphi, \pm 1)=e^{-i \varphi \theta}\left(D_{\theta}-\varphi\right)\left(1+\left(D_{\theta}-\varphi\right)^{2}-\Delta_{\mathbb{B}}\right)^{-1 / 2} e^{i \varphi \theta} \\
& \Gamma_{D_{x} \Lambda}(\varphi, \pm 1)=e^{-i \varphi \theta} D_{x}\left(1+\left(D_{\theta}-\varphi\right)^{2}-\Delta_{\mathbb{B}}\right)^{-1 / 2} e^{i \varphi \theta}
\end{aligned}
$$


REMARK 3.5. Because of the way $\Gamma$ was defined, it is obvious that condition (ii) of Theorem 3.2 can be replaced by

(ii') The matrix $\Gamma_{A}^{N}(m):=\left(\left(\Gamma_{A_{j k}}(m)\right)\right)_{1 \leq j, k \leq N}$ is invertible for all $m \in \mathbf{M}_{S L}$.

Our next and final objective is to find necessary and sufficient conditions for a differential operator with semi-periodic coefficients on $\Omega$ to be Fredholm. Most of the ideas and proofs in what follows are borrowed from [2], $\S \S$ VII.3 and IX.3, where the more general problem of finding differential expressions within reach of a Comparison Algebra is addressed.

Proposition 3.6. Let $L$ be an $M$ th order differential expression on $\mathbb{B}$, with smooth coefficients. The operator $L \Lambda^{M}$, defined initially on the dense subspace $\Lambda^{-M}\left(\mathbf{C}_{0}^{\infty}(\Omega)\right)$, can be extended to a bounded operator $A$ in $\mathscr{L}(\mathscr{H})$. Moreover, we have that $A \in \mathscr{C}_{\mathscr{P}}, \sigma_{A}$ coincides with the principal symbol of $L$ on $\mathbf{W}_{P}$ (points of $\mathbf{M}_{P}$ over $|t|<\infty$ ) and

$$
\begin{array}{r}
\Gamma_{A}(\varphi, \pm 1)=e^{-i \varphi \theta} L\left(1+\left(D_{\theta}-\varphi\right)^{2}-\Delta_{\mathbb{B}}\right)^{-1 / 2} e^{i \varphi \theta}, \\
\left(e^{2 \pi i \varphi}, \pm 1\right) \in \mathbf{M}_{S L} .
\end{array}
$$

Proof. It is easy to see that any $M$ th order differential expression on a compact manifold equals a sum of products of at most $M$ first-order differential expressions. (See, for example, the proof of Proposition VI-3-1 of [2].) It is therefore enough to consider $L$ of the form

$$
L=D_{1} D_{2} \cdots D_{M},
$$

where $D_{j}, j=1, \ldots, M$, are first order expressions. For $M=1$, the proposition is true by Theorem 2.2 and Proposition 3.4.

Using that $\Lambda^{2}=H^{-1}, H=1-\Delta_{\mathbb{R}}-\Delta_{\mathbb{B}}$, it is easy to see that, for $u \in \Lambda^{-2}\left(C_{0}^{\infty}(\Omega)\right)$, and $D_{1}$ and $D_{2}$ first order expressions, we have:

$$
D_{1} D_{2} \Lambda^{2} u=D_{1} \Lambda^{2} D_{2} u+D_{1} \Lambda^{2}\left[H, D_{2}\right] \Lambda^{2} u
$$

The commutator $\left[H, D_{2}\right]$ is a second order expression on $\mathbb{B}$ and can therefore be expressed as a sum of products of at most two first order differential expressions:

$$
\left[H, D_{2}\right]=\sum_{j=1}^{p} F_{j} G_{j} .
$$


This shows that, on the dense subspace $\Lambda^{-2}\left(C_{0}^{\infty}(\Omega)\right), D_{1} D_{2} \Lambda^{2}$ equals the operator

$$
\left(D_{1} \Lambda\right)\left(D_{2}^{*} \Lambda\right)^{*}+\left(D_{1} \Lambda\right) \sum_{j=1}^{p}\left(F_{j}^{*} \Lambda\right)^{*}\left(G_{j} \Lambda\right) \Lambda \in \mathscr{C}_{\mathscr{P}},
$$

where $D^{*}$ denotes the formal adjoint of a differential expression $D$.

Since $\sigma_{\Lambda}=0$, we get:

$$
\sigma_{D_{1} D_{2} \Lambda^{2}}=\sigma_{D_{1} \Lambda} \sigma_{D_{2}^{*} \Lambda}
$$

which, restricted to $\mathbf{W}_{P}$, coincides with the principal symbol of $D_{1}$, $D_{2}$, by Theorem 2.2. It also follows that:

$$
\Gamma_{D_{1} D_{2} \Lambda^{2}}=\Gamma_{D_{1} \Lambda} \Gamma_{D_{2}^{*} \Lambda}^{*}+\Gamma_{D_{1} \Lambda} \sum_{j=1}^{p} \Gamma_{F_{j}^{*} \Lambda}^{*} \Gamma_{G, \Lambda} \Gamma_{\Lambda} .
$$

By Proposition 3.4, we get:

$$
\begin{aligned}
e^{i \varphi \theta} & \Gamma_{D_{1} D_{2} \Lambda^{2}}(\varphi, \pm 1) e^{-i \varphi \theta} \\
& =\left(D_{1} \Lambda_{\varphi}\right)\left(D_{2}^{*} \Lambda_{\varphi}\right)^{*}+D_{1} \Lambda_{\varphi} \sum_{j=1}^{p}\left(F_{j}^{*} \Lambda_{\varphi}\right)^{*}\left(G_{j} \Lambda_{\varphi}\right) \Lambda_{\varphi} \\
& =D_{1} \Lambda_{\varphi}^{2} D_{2}+D_{1} \Lambda_{\varphi}^{2} \sum_{j=1}^{p} F_{j} G_{j} \Lambda_{\varphi}^{2},
\end{aligned}
$$

where $\Lambda_{\varphi}=H_{\varphi}^{-1 / 2}, H_{\varphi}=1+\left(D_{\theta}-\varphi\right)^{2}-\Delta_{\mathbb{B}}$. Since $\left[H, D_{2}\right]$ and $\left[H_{\varphi}, D_{2}\right]$ are equal (as expressions on $\mathbb{B}$ ), we get:

$$
e^{i \varphi \theta} \Gamma_{D_{1} D_{2} \Lambda^{2}}(\varphi, \pm 1) e^{-i \varphi \theta}=D_{1} \Lambda_{\varphi}^{2} D_{2}+D_{1} \Lambda_{\varphi}^{2}\left[H_{\varphi}, D_{2}\right] \Lambda_{\varphi}^{2}=D_{1} D_{2} \Lambda_{\varphi}^{2}
$$

proving the proposition for $L=D_{1} D_{2}$.

Suppose now that the proposition is true for sums of products of at most $M$ first order differential expressions and let $L=D_{1} D_{2} \cdots D_{M+1}$ be a product of first order expressions. Define: $F=D_{1} D_{2}$ and $G=$ $D_{3} \cdots D_{M+1}$. Using the formula

$$
\begin{array}{r}
L \Lambda^{M+1} u=F \Lambda^{2} G \Lambda^{M-1} u+F \Lambda^{2}[H, G] \Lambda^{M+1} u, \\
u \in \Lambda^{-M-1}\left(\mathbf{C}_{0}^{\infty}(\Omega)\right),
\end{array}
$$

the proposition follows for this $L$, by the same argument as above. 
Let $\left\{U_{\beta}\right\}$ be a finite atlas on $\mathbb{B}$ and $\left\{\varphi_{\beta}\right\}$ a subordinate partition of unity, i.e. support $\varphi_{\beta} \subset U_{\beta}$. Let $L$ be a differential operator on $\Omega$, acting on $\mathbb{C}^{N}$-valued functions, locally given on $U_{\beta}$ by

$$
L=\sum_{j=0}^{\widetilde{M}} \sum_{|\alpha| \leq M_{j}} A_{\beta, j, \alpha}(t, x)\left(\frac{1}{i} \frac{\partial}{\partial x}\right)^{\alpha}\left(\frac{1}{i} \frac{\partial}{\partial t}\right)^{j},
$$

where

$$
\left(\frac{1}{i} \frac{\partial}{\partial x}\right)^{\alpha}:=\left(-i \frac{\partial}{\partial x_{1}}\right)^{\alpha_{1}} \cdots\left(-i \frac{\partial}{\partial x_{n}}\right)^{\alpha_{n}}, \quad \text { for } \alpha \in \mathbb{N}^{n}
$$

and $|\alpha|=\alpha_{1}+\cdots+\alpha_{n}$. We will say that $L$ has semi-periodic coeffcients if the matrices

$$
\widetilde{A}_{\beta, j, \alpha}(t, x):=\varphi_{\beta}(x) A_{\beta, j, \alpha}(t, x),
$$

regarded as functions on $\Omega$, have as entries functions of the type (28). It is easy to see that this definition is independent of the choice of atlas on $\mathbb{B}$. We want to decide when

$$
L: H^{M}\left(\Omega, \mathbb{C}^{N}\right) \rightarrow L^{2}\left(\Omega, \mathbb{C}^{N}\right)
$$

is a Fredholm operator, assuming that $L$ has semi-periodic coefficients. Here $M$ denotes the order of $L, M=\max \left\{M_{j}+j, j=\right.$ $1, \ldots, \widetilde{M}\}$.

We also denote by $\Lambda$ the operator $\Lambda \otimes I_{N}$ on $\mathscr{L}\left(L^{2}\left(\Omega, \mathbb{C}^{N}\right)\right)$, where $I_{N}$ denotes the $N \times N$ identity matrix. Since $\Lambda$ commutes with $\frac{\partial}{\partial t}$ and $L=\sum L_{\beta}$, for $L_{\beta}:=\varphi_{\beta} L$, we get:

$$
L \Lambda^{M}=\sum_{\beta, j, \alpha}(t, x)\left(\frac{1}{i} \frac{\partial}{\partial x}\right)^{\alpha} \Lambda^{|\alpha|}\left(\frac{1}{i} \frac{\partial}{\partial t}\right)^{j} \Lambda^{j} \Lambda^{M-|\alpha|-j} .
$$

After multiplying $\left(\frac{1}{i} \frac{\partial}{\partial x}\right)^{\alpha}$ above by $\chi_{\beta, j, \alpha} \in \mathbf{C}_{0}^{\infty}\left(U_{\beta}\right), \chi_{\beta, j, \alpha}(x)=1$ for $x$ in the support of $\widetilde{A}_{\beta, j, \alpha}$, we still get the same operator and $\chi_{\beta, j, \alpha}(x)\left(\frac{1}{i} \frac{\partial}{\partial x}\right)^{\alpha}$ is now a differential expression defined on $\mathbb{B}$. We can therefore apply Proposition 3.6 and conclude that $L \Lambda^{M} \in \mathscr{C}_{\mathscr{P}}^{N}$. Using, moreover, that $\sigma_{\Lambda^{M-|\alpha|-\jmath}}=0$ for $|\alpha|+j<M$, we get:

$$
\sigma_{L \Lambda^{M}}(t, x ; \tau, \xi)=\sum_{\beta} \sum_{|\alpha|+j=M} \tilde{A}_{\beta, j, \alpha}(t, x) \xi^{\alpha} \tau^{j}, \quad|t|<\infty .
$$

The right-hand side of the previous equation coincides with the principal symbol of $L$ restricted to the co-sphere bundle of $\Omega$. Invertibility 
of the $\sigma$-symbol is therefore equivalent to uniform ellipticity of $L$, by Remark 2.3.

The operator-valued symbol $\Gamma_{L \Lambda^{M}}$ is also given by Proposition 3.6 (and Proposition 3.4):

$$
\begin{aligned}
e^{-i \varphi \theta} & \Gamma_{L \Lambda^{M}}(\varphi, \pm 1) e^{i \varphi \theta} \\
& =\sum_{\beta, j, \alpha} \widetilde{A}_{\beta, j, \alpha}^{ \pm}(\theta, x)\left(\frac{1}{i} \frac{\partial}{\partial x}\right)^{\alpha}\left(\frac{1}{i} \frac{\partial}{\partial \theta}-\varphi\right)^{j} \Lambda_{\varphi}^{M},
\end{aligned}
$$

where we have used that $\Lambda_{\varphi}$ and $\frac{\partial}{\partial \theta}$ commute. We have denoted by $\widetilde{A}_{\beta, j, \alpha}^{ \pm}$the $2 \pi$-periodic continuous functions such that

$$
\widetilde{A}_{\beta, j, \alpha}(t, x)-\chi_{+}(t) \tilde{A}_{\beta, j, \alpha}^{+}(t, x)-\chi_{-}(t) \tilde{A}_{\beta, j, \alpha}^{-}(t, x) \in \mathbf{C O}(\Omega) .
$$

(See (28).)

Let $L_{\beta}^{ \pm}(\varphi)$ denote the differential expressions on $\mathbb{S}^{1} \times \mathbb{B}$

$$
L_{\beta}^{ \pm}(\varphi):=\sum_{j=0}^{\widetilde{M}} \sum_{|\alpha| \leq M,}+\widetilde{A}_{\beta, j, \alpha}^{ \pm}(\theta, x)\left(\frac{1}{i} \frac{\partial}{\partial x}\right)^{\alpha}\left(\frac{1}{i} \frac{\partial}{\partial \theta}-\varphi\right)^{j}
$$

and define the operator

$$
L^{ \pm}(\varphi):=\sum_{\beta} L_{\beta}^{ \pm}(\varphi): H^{M}\left(\mathbb{S}^{1} \times \mathbb{B}, \mathbb{C}^{N}\right) \rightarrow L^{2}\left(\mathbb{S}^{1} \times \mathbb{B}, \mathbb{C}^{N}\right)
$$

Since $\Lambda_{\varphi}$ is an isomorphism from

$$
L^{2}\left(\mathbb{S}^{1} \times \mathbb{B}, \mathbb{C}^{N}\right) \text { onto } H^{M}\left(\mathbb{S}^{1} \times \mathbb{B}, \mathbb{C}^{N}\right),
$$

the above considerations, together with Theorem 3.2 and Remark 3.5 prove the following theorem.

THEOREM 3.7. Let $L$ denote an $M$ th order differential operator on $\Omega$ of the form (38), with continuous semi-periodic coefficients, and let $L^{ \pm}(\varphi)$ denote the differential operators on $S^{1} \times \mathbb{B}$ defined in (39). Then

$$
L: H^{M}\left(\Omega, \mathbb{C}^{N}\right) \rightarrow L^{2}\left(\Omega, \mathbb{C}^{N}\right)
$$

is Fredholm if and only if $L$ is uniformly elliptic and $L^{ \pm}(\varphi)$ are invertible for all $\varphi \in[0,1]$. 


\section{REFERENCES}

[1] M. Breuer and H. O. Cordes, On Banach algebras with $\sigma$-symbol, part 2, J. Math. Mech., 14 (1965), 299-314.

[2] H. O. Cordes, Spectral theory of linear differential operators and comparison algebras, London Math. Soc. Lecture Notes Vol. 76, Cambridge University Press 1987.

[3] _ On the two-fold symbol chain of a $C^{*}$-algebra of singular integral operators on a polycylinder, Revista Mat. Iberoamericana, 2 (1986), 215-234.

[4] _ Elliptic pseudo-differential operators, an abstract theory, Springer Lecture Notes in Math., Vol. 756, Berlin, Heildelberg, New York 1979.

[5] H. O. Cordes and S. T. Melo, An algebra of singular integral operators with kernels of bounded oscillation, and application to periodic differential operators, J. Differential Equations, 75 (1988), 216-278.

[6] R. Lockhart and R. McOwen, Elliptic differential operators on noncompact manifolds, Annali della Scuola Normale Superiore di Pisa, IV-XII (1985), 409-447.

[7] S. T. Melo, Comparison Algebras with Periodic Symbols, Thesis, Berkeley 1988.

[8] C. H. Taubes, Gauge theory on asymptotically periodic 4-manifolds, J. Differential Geometry, 25 (1987), 363-430.

Received September 26, 1988. This work is part of my thesis at Berkeley, under the advise of Professor H. O. Cordes, to whom I wish to express my deepest thanks. I was supported by the Brazilian Government, through CAPES and UFPE.

Universidade Fed. de Pernambuco

50739 RECIFE PE, BRAZIL 


\section{PACIFIC JOURNAL OF MATHEMATICS EDITORS}

\author{
V. S. VARADARAJAN \\ (Managing Editor) \\ University of California \\ Los Angeles, CA 90024-1555-05 \\ Herbert Clemens \\ University of Utah \\ Salt Lake City, UT 84112 \\ Thomas EnRIght \\ University of California, San Diego \\ La Jolla, CA 92093
}

R. FINN

Stanford University

Stanford, CA 94305

HeRmanN FlaschKa

University of Arizona

Tucson, AZ 85721

VAughan F. R. Jones

University of California

Berkeley, CA 94720

Steven Kerckhoff

Stanford University

Stanford, CA 94305

\section{C. Moore}

University of California

Berkeley, CA 94720

Martin ScharlemanN

University of California

Santa Barbara, CA 93106

Harold Stark

University of California, San Diego

La Jolla, CA 92093

\section{ASSOCIATE EDITORS}
R. ARENS
E. F. BECKENBACH
B. H. NEUMANN
F. WoLF
K. YoshidA (1906-1982)
(1904-1989)

\section{SUPPORTING INSTITUTIONS}

UNIVERSITY OF ARIZONA
UNIVERSITY OF BRITISH COLUMBIA
CALIFORNIA INSTITUTE OF TECHNOLOGY
UNIVERSITY OF CALIFORNIA
MONTANA STATE UNIVERSITY
UNIVERSITY OF NEVADA, RENO
NEW MEXICO STATE UNIVERSITY
OREGON STATE UNIVERSITY

\author{
UNIVERSITY OF OREGON \\ UNIVERSITY OF SOUTHERN CALIFORNIA \\ STANFORD UNIVERSITY \\ UNIVERSITY OF HAWAII \\ UNIVERSITY OF TOKYO \\ UNIVERSITY OF UTAH \\ WASHINGTON STATE UNIVERSITY \\ UNIVERSITY OF WASHINGTON
}

The Supporting Institutions listed above contribute to the cost of publication of this Journal, but they are not owners or publishers and have no responsibility for its content or policies.

Mathematical papers intended for publication in the Pacific Journal of Mathematics should be in typed form or offset-reproduced (not dittoed), double spaced with large margins. Please do not use built up fractions in the text of the manuscript. However, you may use them in the displayed equations. Underline Greek letters in red, German in green, and script in blue. The first paragraph must be capable of being used separately as a synopsis of the entire paper. In particular it should contain no bibliographic references. Please propose a heading for the odd numbered pages of less than 35 characters. Manuscripts, in triplicate, may be sent to any one of the editors. Please classify according to the 1980 Mathematics Subject Classification (1985 Revision) scheme which can be found in the December index volumes of Mathematical Reviews. Supply name and address of author to whom proofs should be sent. All other communications should be addressed to the managing editor, or Elaine Barth, University of California, Los Angeles, California 90024-1555-05.

There are page-charges associated with articles appearing in the Pacific Journal of Mathematics. These charges are expected to be paid by the author's University, Government Agency or Company. If the author or authors do not have access to such Institutional support these charges are waived. Single authors will receive 50 free reprints; joint authors will receive a total of 100 free reprints. Additional copies may be obtained at cost in multiples of 50 .

The Pacific Journal of Mathematics (ISSN 0030-8730) is published monthly. Regular subscription rate: $\$ 190.00$ a year (12 issues). Special rate: $\$ 95.00$ a year to individual members of supporting institutions.

Subscriptions, orders for numbers issued in the last three calendar years, and changes of address should be sent to Pacific Journal of Mathematics, P.O. Box 969, Carmel Valley, CA 93924, U.S.A. Old back numbers obtainable from Kraus Periodicals Co., Route 100, Millwood, NY 10546.

The Pacific Journal of Mathematics at P.O. Box 969, Carmel Valley, CA 93924 (ISSN 0030-8730) is published monthly. Second-class postage paid at Carmel Valley, California 93924, and additional mailing offices. Postmaster: send address changes to Pacific Journal of Mathematics, P.O. Box 969, Carmel Valley, CA 93924.

PUBLISHED BY PACIFIC JOURNAL OF MATHEMATICS, A NON-PROFIT CORPORATION

Copyright (C) 1990 by Pacific Journal of Mathematics 


\section{Pacific Journal of Mathematics}

\section{Vol. 146, No. 2 December, 1990}

David Fenimore Anderson, David Earl Dobbs, Paul M. Eakin, Jr. and

William James Heinzer, On the generalized principal ideal theorem and Krull domains .................................... 201

Richard C. Bradley, On $\rho$-mixing except on small sets $\ldots \ldots \ldots \ldots \ldots 217$

David H. Collingwood and Brad Shelton, A duality theorem for extensions

of induced highest weight modules $\ldots \ldots \ldots \ldots \ldots \ldots \ldots \ldots \ldots \ldots 27$

Gerd H. Fricke and John Albert Fridy, Sequence transformations that guarantee a given rate of convergence $\ldots . \ldots \ldots \ldots \ldots \ldots \ldots . \ldots . \ldots . \ldots 239$

Michael Justin Heumos and Stephen James Rallis, Symplectic-Whittaker

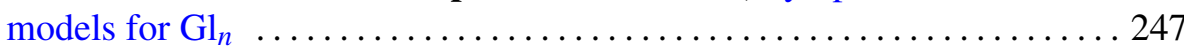

Severino Toscano do Rego Melo, A comparison algebra on a cylinder with

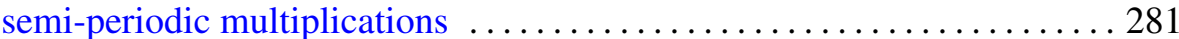

Daniel Pecker, On the elimination of algebraic inequalities ........... 305

Geoffrey Lynn Price, The $C^{*}$-algebras generated by pairs of semigroups of isometries satisfying certain commutation relations $\ldots \ldots \ldots \ldots \ldots \ldots 315$

John Kurt Sauter, Jr., Isomorphisms among monodromy groups and

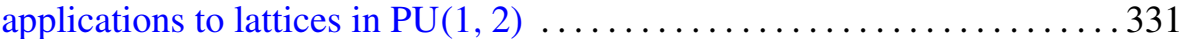

Shoji Yokura, A formula for Segre classes of singular projective varieties . . 385 